\title{
Simplified Space-Mapping Approach to Enhancement of Microwave Device Models
}

\author{
Qingsha S. Cheng, Slawomir Koziel, John W. Bandler \\ Simulation Optimization Systems Research Laboratory, Department of Electrical and Computer \\ Engineering, McMaster University, Hamilton, Ontario L8S 4K1, Canada
}

Received 5 August 2005; accepted 28 October 2005

\begin{abstract}
In this article, we present advances in microwave and RF device modeling exploiting the space mapping (SM) technology. New SM-based modeling techniques are proposed that are easy to implement entirely in the Agilent ADS framework. A simplified SM-based model description is discussed. Using a two-section transformer example, we show how the modeling accuracy is affected by the model flexibility. Tables, diagrams, and flowcharts are developed to help in understanding the concepts. This makes the SM modeling concept available to engineers through widely used commercial software. Our approach permits the creation of library models that can be used for model enhancement of microwave elements. Frequency-interpolation techniques are discussed and implemented. A set of four different SM-based models is presented along with corresponding implementations in the ADS schematic for a microstrip right-angle bend and a microstrip shaped $\mathrm{T}$-junction. We use a three-section transformer to illustrate the implementation procedure in full details. We apply the technique to a more complicated HTS filter modeling problem. Fine-model data is obtained from Sonnet's em. We discuss the relation between the model complexity and accuracy as well as further improvement of the model. () 2006 Wiley Periodicals, Inc. Int J RF and Microwave CAE 16: 518-535, 2006.
\end{abstract}

Keywords: CAD; optimization; EM modeling; microstrip modeling; microwave filters; space mapping; surrogate modeling

\section{INTRODUCTION}

Space mapping (SM) technology [1-3] addresses the issue of reducing unnecessary time-consuming fullwave EM simulations of microwave structures in device modeling problems and design optimization. SM assumes the existence of "fine" and "coarse" models. The "fine" model may be a CPU-intensive EM sim-

Correspondence to: John W. Bandler; email: bandler@mcmaster.ca.

This work was supported in part by the Natural Sciences and Engineering Research Council of Canada under grant numbers OGP0007239, STGP269760, and STGP269889, and by Bandler Corporation.

DOI 10.1002/mmce.20172

Published online 20 July 2006 in Wiley InterScience (www. interscience.wiley.com). ulator that provides high accuracy. The "coarse" model is a simplified representation, typically an equivalent circuit with empirical formulas. Modeling implementations of SM exploit the computational efficiency of coarse models and the accuracy of corresponding fine models so as to realize fast and accurate (enhanced) models valid in a wide range of the parameter spaces. Space derivative mapping [4], the so-called generalized SM [5] and SM-based neuromodeling $[6,7]$ are three approaches. Space derivative mapping uses the Jacobians of the fine and coarse models. The generalized SM approach exploits input and frequency mapping, and multiple SM. These are simple methods, however, they only use the so-called input space mapping and frequency scaling, which may not provide enough flexibility for more compli- 


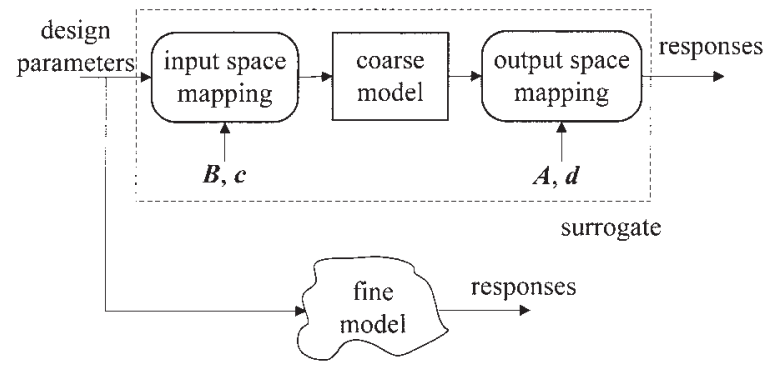

Figure 1. Illustration of the SM-based model.

cated problems. SM-based neuromodeling establishes the mapping using artificial neural networks. This approach is reliable; however, it cannot be directly implemented in ADS and requires specialized neural network software. A review of different SM methods is presented in [3].

It is desirable to engineers that SM-based models can be set up and used in connection with (or even within) available commercial software. Our article explores this possibility. We consider a family of models that is implemented entirely in the Agilent ADS [8] framework. The family involves only certain combinations of input and output space mapping. The models can be used as library models for corresponding microwave elements and hence for direct CAD, optimization, and yield design.

Figure 1 shows the configuration of the SM-based model and fine model in training or testing. The mapping parameters $\boldsymbol{B}, \boldsymbol{c}, \boldsymbol{A}$, and $\boldsymbol{d}$ are calibrated such that multiple sets of responses of the SM-based model match those of the fine model simultaneously.

\section{MODEL DESCRIPTION}

Let $\boldsymbol{R}_{f}: X_{f} \rightarrow R^{m}$ and $\boldsymbol{R}_{c}: X_{c} \rightarrow R^{m}$ denote the fine and coarse model response vectors, where $X_{f} \subseteq R^{n}$ and $X_{c}$ $\subseteq R^{n}$ are design variable domains of the fine and coarse models, respectively. For example, $R_{f}(\boldsymbol{x})$ and $\boldsymbol{R}_{c}(\boldsymbol{x})$ may represent magnitudes of a microwave filter transfer function at $m$ chosen frequencies.

We denote by $X_{R} \subseteq X_{f}$ the region of interest in which we want enhanced matching between the surrogate and the fine model. We assume that $X_{R}$ is an $n$-dimensional interval in $R^{n}$ with center at reference point $\boldsymbol{x}^{0} \in R^{n}$ :

$$
X_{R}=\left[\boldsymbol{x}^{0}-\boldsymbol{\delta}, \boldsymbol{x}^{0}+\boldsymbol{\delta}\right]
$$

where $\boldsymbol{\delta}=\left[\delta_{1} \ldots \delta_{n}\right]^{T} \in R_{+}^{n}$ determines the size of $X_{R}$. We use $X_{R}\left(\boldsymbol{x}^{0}, \boldsymbol{\delta}\right)$ to denote the region of interest defined by $\boldsymbol{x}^{0}$ and $\boldsymbol{\delta}$.

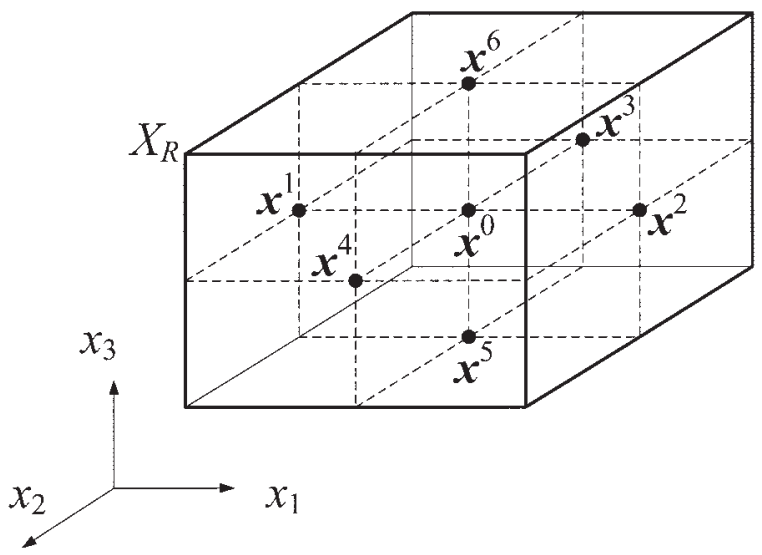

Figure 2. The region of interest $X_{R}$ and base set $X_{B}$ for $n=3[5]$.

The number of fine-model evaluations used to construct a surrogate should be small, since we assume that each evaluation is expensive. But, we have to account for the dimension of the design variable space. In this work, we use the set of evaluation points (also called the base set) denoted by $X_{B}$, to consist of $2 n+1$ points (Figure 2), where $n=\operatorname{dim}\left(X_{f}\right)$. We have

$$
X_{B}=\left\{\boldsymbol{x}^{0}, \boldsymbol{x}^{1}, \ldots, \boldsymbol{x}^{2 n}\right\}
$$

where $x^{0}$ is the reference point, and

$$
\boldsymbol{x}^{j}=\boldsymbol{x}^{0}+(-1)^{j} \delta_{\lceil j / 2\rceil} \cdot \boldsymbol{u}_{\lceil j / 2\rceil}, \quad j=1, \ldots, 2 n,
$$

where $\boldsymbol{u}_{k}=\left[\begin{array}{lllllll}0 & \ldots & 0 & 1 & 0 & \ldots & 0\end{array}\right]^{T}$ is a unit vector with 1 at the $k^{\text {th }}$ position; $\delta_{k}$ is the size of the region $X_{R}$ along the $k^{\text {th }}$ axis. This distribution of points is called the star distribution [5]. In general, the base set is not limited to the star distribution.

We simplify the description in [9]. We define a generic surrogate model $\boldsymbol{R}_{s}: X_{f} \times M_{m \times m} \times M_{n \times n} \times$ $M_{n \times 1} \times M_{m \times 1} \rightarrow R^{m}$ as

$$
\boldsymbol{R}_{s}(\boldsymbol{x}, \boldsymbol{A}, \boldsymbol{B}, \boldsymbol{c}, \boldsymbol{d})=\boldsymbol{A} \cdot \boldsymbol{R}_{c}(\boldsymbol{B} \cdot \boldsymbol{x}+\boldsymbol{c})+\boldsymbol{d}
$$

with matrices $\boldsymbol{A}=\operatorname{diag}\left\{a_{1}, \ldots, a_{m}\right\}, \quad \boldsymbol{B} \in M_{n \times n}$, $c \in M_{n \times 1}$, and $\boldsymbol{d} \in M_{m \times 1}\left(M_{k \times l}\right.$ denotes the set of $k \times l$ real matrices). $\boldsymbol{B}$ and $\boldsymbol{c}$ account for the input mapping [5]. $\boldsymbol{A}$ and $\boldsymbol{d}$ provide an output mapping [10].

We consider four SM-based surrogate models $\boldsymbol{R}_{s i}$ : $X_{f} \rightarrow R^{m}, i=1,2,3,4$. All the models are defined as

$$
\boldsymbol{R}_{s i}(\boldsymbol{x})=\boldsymbol{R}_{s}(\boldsymbol{x}, \overline{\boldsymbol{A}}, \overline{\boldsymbol{B}}, \overline{\boldsymbol{c}}, \overline{\boldsymbol{d}})
$$

for $i=1,2,3,4$, where matrices $\overline{\boldsymbol{A}}, \overline{\boldsymbol{B}}, \overline{\boldsymbol{c}}$, and $\overline{\boldsymbol{d}}$ found using the parameter extraction 


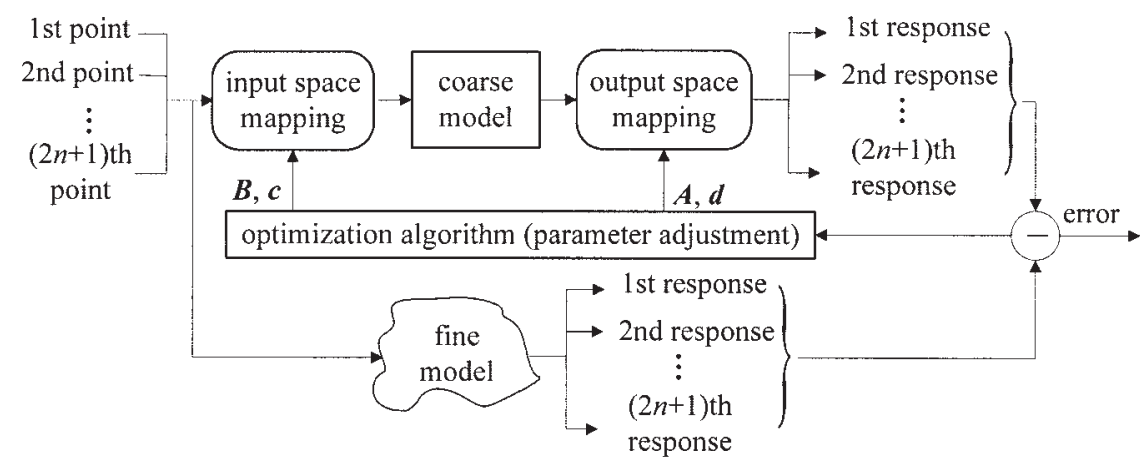

Figure 3. Illustration of the SM-based model optimization (parameter extraction).

$(\bar{A}, \bar{B}, \bar{c}, \bar{d})$

$$
=\arg \min _{(A, B, c, d)} \sum_{k=0}^{2 n}\left\|\boldsymbol{R}_{f}\left(\boldsymbol{x}^{k}\right)-\boldsymbol{R}_{s}\left(\boldsymbol{x}^{k}, \boldsymbol{A}, \boldsymbol{B}, \boldsymbol{c}, \boldsymbol{d}\right)\right\|
$$

This procedure may be visualized as in Figure 3. The optimization algorithm uses the error between the fine and the surrogate to adjust $\boldsymbol{B}, \boldsymbol{c}, \boldsymbol{A}$, and $\boldsymbol{d}$.

Parameter extraction (6) for model $\boldsymbol{R}_{s 1}$ is subject to the constraints $\boldsymbol{A}=\boldsymbol{I}_{n}$, and $\boldsymbol{d}=\mathbf{0}_{m \times 1}\left(\boldsymbol{I}_{n}\right.$ is an $n \times n$ identity matrix, $\mathbf{0}_{m \times 1}$ is the zero $M_{m \times 1}$ matrix,). For model $\boldsymbol{R}_{s 2}$ the constraint is $\boldsymbol{d}=\mathbf{0}_{m \times 1}$. For model $\boldsymbol{R}_{s 3}$ the constraint is $\boldsymbol{A}=\boldsymbol{I}_{n}$. In the case of model $\boldsymbol{R}_{s 4}$, the parameter extraction is performed without constraints. We summarize this in Table I, in which we list five models/surrogates with different flexibilities or degrees of freedom. The constraint limits the number of mapping variables of the models. The less constrained the model, the more it gains complexity and accuracy. For $\boldsymbol{R}_{s 1}$, we use a linear input mapping for the design variables. For $\boldsymbol{R}_{s 2}, \boldsymbol{R}_{s 3}$, and $\boldsymbol{R}_{s 4}$, complexity is gradually added using output mapping parameters $\boldsymbol{A}$ and/or $\boldsymbol{d}$ to calibrate the responses. A discussion of other properties and limitations of this approach is provided in section $\mathrm{V}$.

Figure 4 shows the SM modeling flowchart. We start by selecting the fine and coarse models and deciding on the mapping parameters $\boldsymbol{B}, \boldsymbol{c}, \boldsymbol{A}, \boldsymbol{d}$. We generate $2 n+1$ base points and multiple random test

TABLE I. Constraints for Different Models

\begin{tabular}{lll}
\hline Model & \multicolumn{1}{c}{ Constraint } & PE Parameters \\
\hline $\boldsymbol{R}_{c}$ & $\boldsymbol{B}=\boldsymbol{I}_{n}, \boldsymbol{c}=\mathbf{0}_{n \times 1}$, & N/A \\
& $\boldsymbol{A}=\boldsymbol{I}_{m}$, and $\boldsymbol{d}=\mathbf{0}_{m \times 1}$ & \\
$\boldsymbol{R}_{s 1}$ & $\boldsymbol{A}=\boldsymbol{I}_{m}$, and $\boldsymbol{d}=\mathbf{0}_{m \times 1}$ & $\boldsymbol{B}$ and $\boldsymbol{c}$ \\
$\boldsymbol{R}_{s 2}$ & $\boldsymbol{d}=\mathbf{0}_{m \times 1}$ & $\boldsymbol{B}, \boldsymbol{c}$, and $\boldsymbol{A}$ \\
$\boldsymbol{R}_{s 3}$ & $\boldsymbol{A}=\boldsymbol{I}_{m}$ & $\boldsymbol{B}, \boldsymbol{c}$, and $\boldsymbol{d}$ \\
$\boldsymbol{R}_{s 4}$ & unconstrained & $\boldsymbol{B}, \boldsymbol{c}, \boldsymbol{A}$, and $\boldsymbol{d}$ \\
\hline
\end{tabular}

points (test set). All the (base and test) points are simulated using the fine model. We perform parameter extraction using $2 n+1$ base points simultaneously. We test the SM-based surrogate model using the test set. Figure 5 indicates the test configuration of the SM-based surrogate model and the fine model.

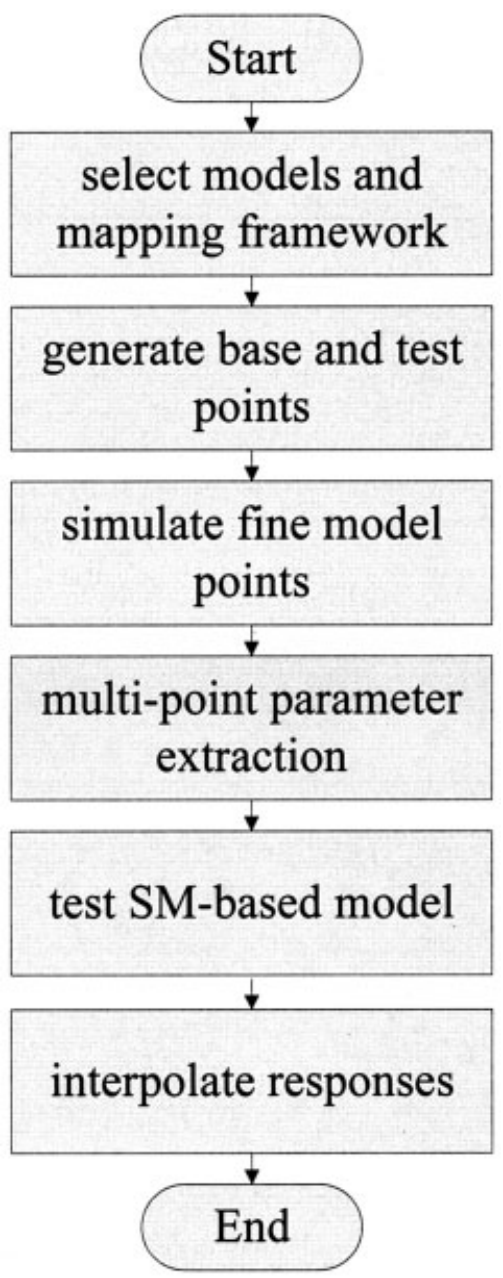

Figure 4. Flowchart of space-mapping enhanced modeling. 


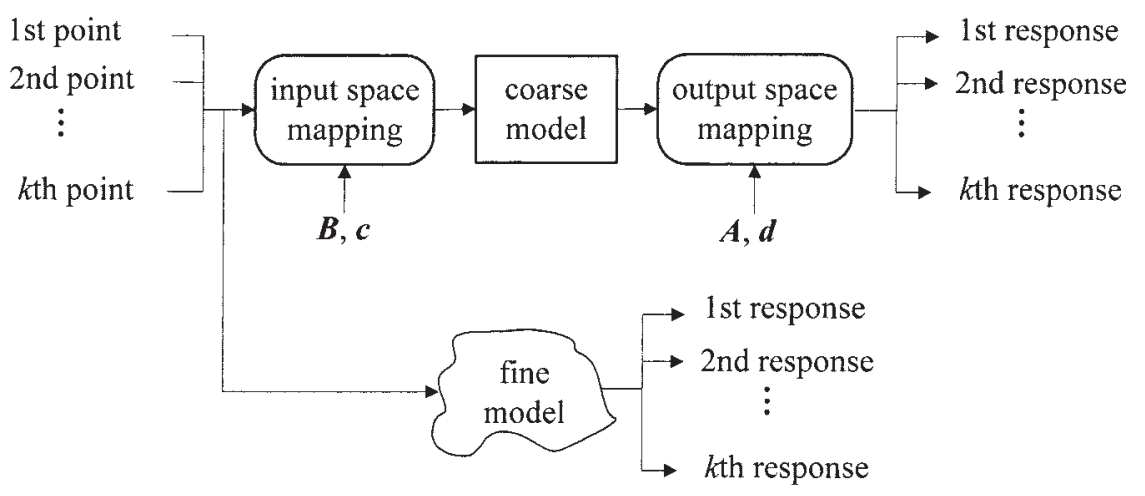

Figure 5. Illustration of the configuration of the fine and the SM-based model for the test phase. A set of (test) points is used to compare the responses between the SM-enhanced model and the fine model.

Before using the SM-based model, we interpolate the responses for arbitrary frequency values. For a frequency point $\omega \in\left[\omega_{j}, \omega_{j+1}\right]$, where $\omega_{j}$ and $\omega_{j+1}$ are two adjacent sample points and $j=1, \ldots, m$, the interpolated response is given by

$$
R_{s i}(\omega)=\mu R_{s i}\left(\omega_{j}\right)+(1-\mu) R_{s i}\left(\omega_{j+1}\right)
$$

where

$$
\mu=\frac{\omega_{j+1}-\omega}{\omega_{j+1}-\omega_{j}} .
$$

The other way of interpolation is formulated as

$$
\begin{aligned}
R_{s i}(\omega)=\left[\mu a_{j}+(1-\mu)\right. & \left.a_{j+1}\right] R_{c}(\omega) \\
& +\mu d_{j}+(1-\mu) d_{j+1}
\end{aligned}
$$

where $a_{j}$ and $d_{j}$ are the $j^{\text {th }}$ components of the diagonal element of matrix $\boldsymbol{A}$ and vector $\boldsymbol{d}$, respectively, $\mu$ is given by (8), and $R_{c}(\omega)$ is the (input-mapped coarse model) frequency response at $\omega$.

The method based on (9) takes full advantage of the (input-mapped) coarse model. Since the (inputmapped) coarse model is smooth in most cases, the linear interpolation (9) is smoother than (7). A comparison is given in section IV.

We now use a two-section transformer to show how increasing the flexibility of the model improves its accuracy. We consider a capacitively loaded 10:1 impedance transformer. The "coarse" model and the "fine" model are an ideal two-section transmission line (TL) and a capacitively-loaded TL with capacitors $C_{1}=C_{2}=$ $C_{3}=10 \mathrm{pF}$. See Figure 6. The electrical lengths $L_{1}$ and $L_{2}$ at $1 \mathrm{GHz}$ are chosen as design parameters. The frequency range is $0.5 \mathrm{GHz} \leq \omega \leq 1.5 \mathrm{GHz}$ in steps of $0.05 \mathrm{GHz}$. The reference point $\boldsymbol{x}^{0}=\left[\begin{array}{ll}75^{\circ} & 80^{\circ}\end{array}\right]^{T}$. The characteristic impedances are kept fixed at the optimal values $Z_{1}=2.2361 \Omega$ and $Z_{2}=4.4721 \Omega$. The fine and coarse models are implemented in Matlab.

Figure 7 shows the coarse-model error surface plot. Figures 8 and 9 show the $\boldsymbol{R}_{s 1}$ and $\boldsymbol{R}_{s 2}$ model error surface plots. $\boldsymbol{R}_{s 2}$ with increased parameters has a higher accuracy than $\boldsymbol{R}_{s 1}$.

\section{ADS IMPLEMENTATION}

Unlike the design optimization framework [11], our modeling implementation uses several points concurrently for parameter extraction. Instead of using the SNP $[8,11]$ file, we uses Agilent's S2PMDIF (multidimensional 2-Port $S$-parameter file) component in the schematic. It can read and "look up" or "interpolate" responses with regard to the desired parameters in measurement data interchange format (MDIF). The sets (base set or test set) of desired parameters are saved in a database file. They can be read by the data

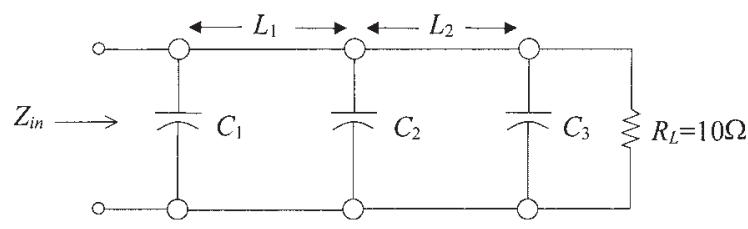

(a)

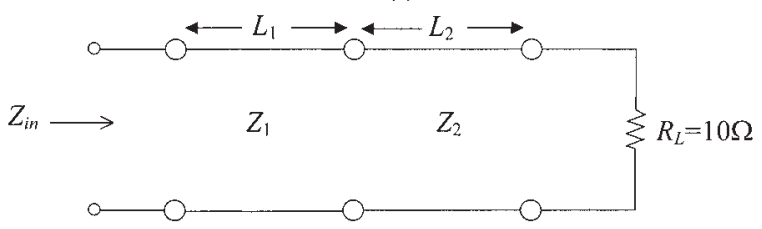

(b)

Figure 6. Two-section capacitively loaded 10:1 impedance transformer [3, 17]: (a) fine model; (b) coarse model. 


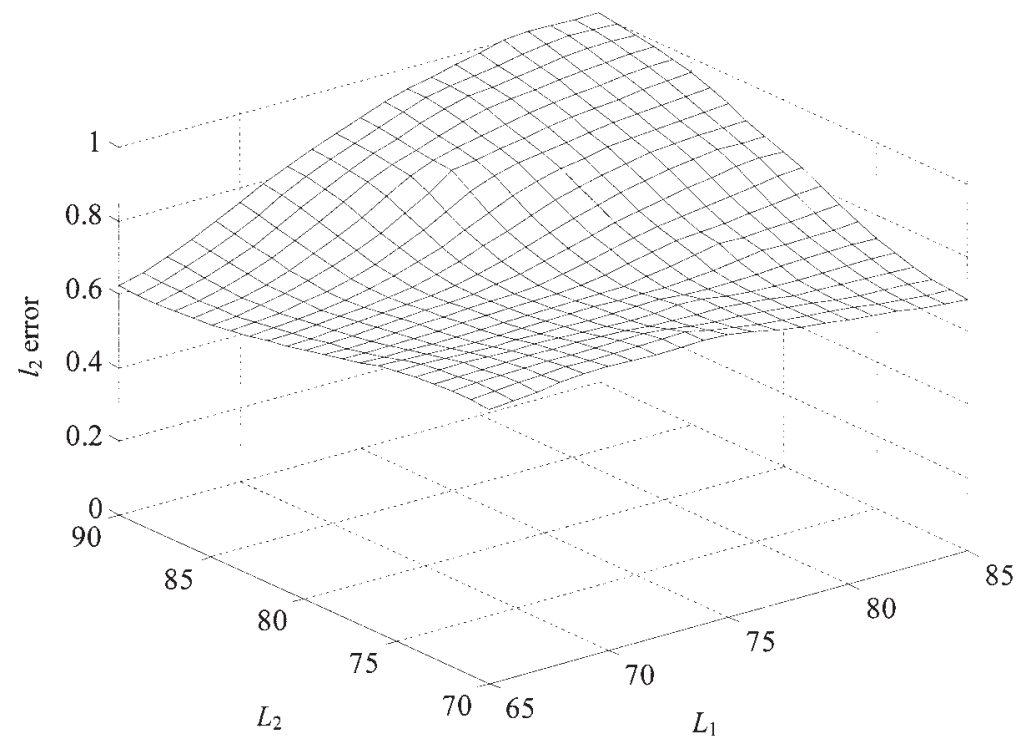

Figure 7. $l_{2}$ error vs. design variables for the two-section transformer (coarse model $\boldsymbol{R}_{c}$ ).

access component (DAC). We can use the parameter sweep component to iterate through the parameters in the database file. Figure 10 shows the ADS schematic setup of reading the fine-model responses for multiple points in a single file. Using those components, a schematic can be set up to match surrogate responses to fine-model responses.

\section{A. ADS Schematic-Modeling Framework for SM with Two-Ports}

Step 1: Set up the fine-model simulator (for example, Sonnet em [12]); execute simulations using the base set of points; save the responses in MDIF format.

Step 2: Set up the coarse model in the ADS schematic; set up the SM surrogate models as (4) (the input and output mapping settings are shown in Figure 11); set up the equality constraints in Table I to represent different models by fixing certain mapping parameters; set up the optimization and goals components (matching the magnitude of $S_{11}$, and so on); set up the parameter sweep to sweep the base set; set up the DAC component to read the base set point values from the database file; set up S2PMDIF to read the fine-model responses.

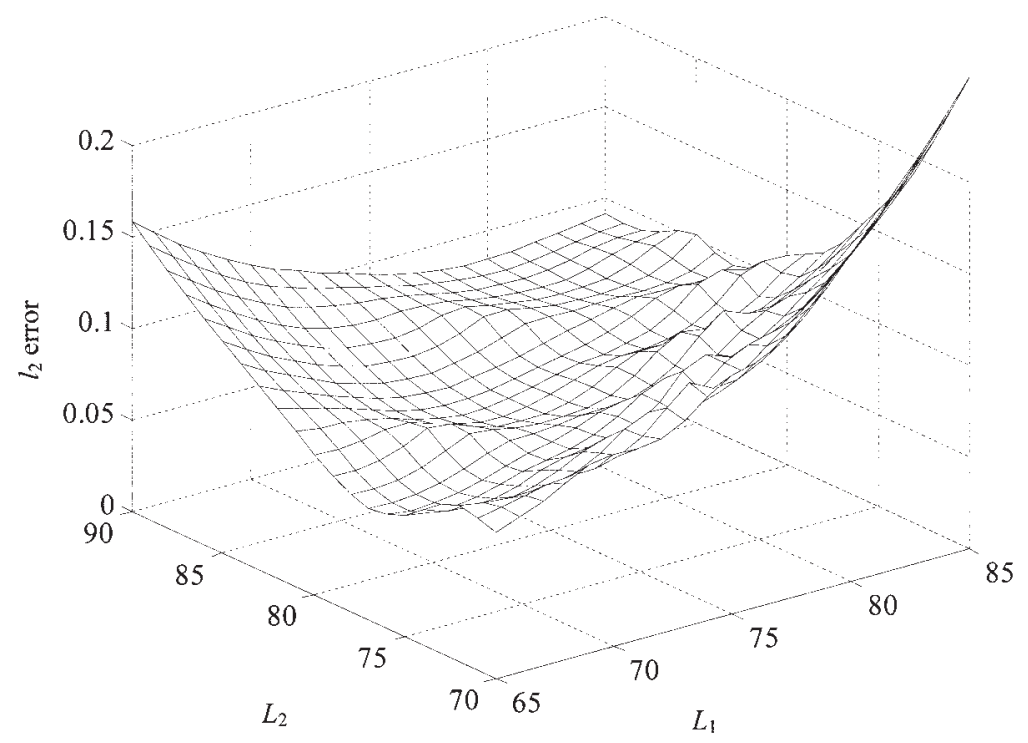

Figure 8. $l_{2}$ error vs. design variables for the two-section transformer (surrogate $\boldsymbol{R}_{s 1}$ ). 


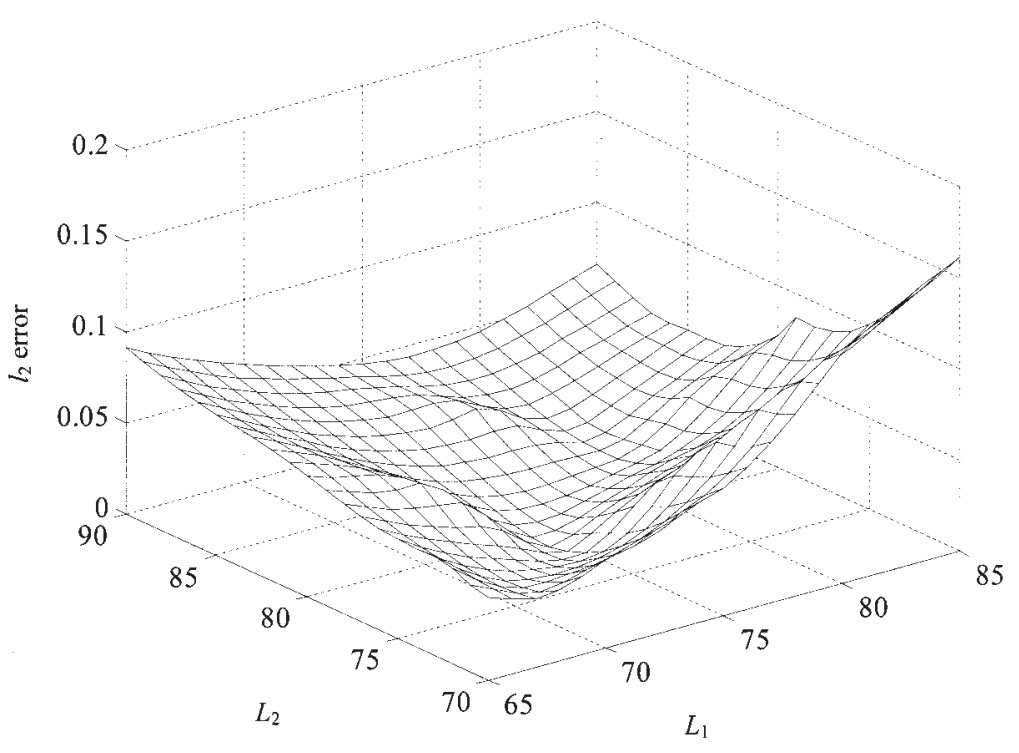

Figure 9. $l_{2}$ error vs. design variables for the two-section transformer (surrogate $\boldsymbol{R}_{s 2}$ ).

Step 3: Calibrate the coarse model (SM-based surrogate) with regard to the mapping parameters $\boldsymbol{B}$, $\boldsymbol{c}, \boldsymbol{A}$, and $\boldsymbol{d}$ to match the fine model at all base points using the ADS optimization algorithm (this is the parameter-extraction step); update the model. Step 4: Deactivate the optimization and goals. Step 5: Generate random test points and save them in a test set database file; set up the fine-model simulator; simulate using the test set; save the responses in a MDIF file.

Step 6: Revise the parameter sweep, DAC, and S2PMDIF components in the schematic to load the test set of values and responses.

Step 7: Simulate and compare the responses.
Step 8: Interpolate the responses with regard to frequency.

Comment. In step 3, if a good match cannot be achieved, an additional operation may be needed, for example, increasing flexibility. In step 7, if the test is not satisfactory, we may require more data. These points are discussed in section $\mathrm{V}$.

Next we can use the surrogate after deleting or deactivating the parameter sweep, DAC, and S2PMDIF with its terminals from the schematic. We can apply the parameters to the surrogate as to a new model. The model is easily switched between $\boldsymbol{R}_{c}, \boldsymbol{R}_{s 1}$,

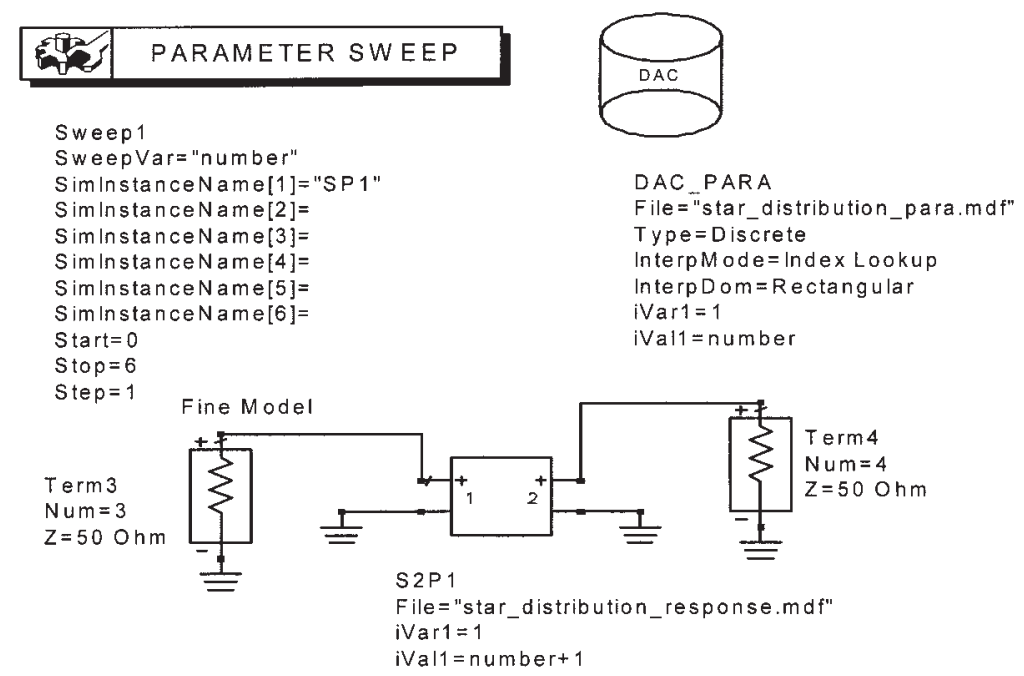

Figure 10. ADS schematic setup of the fine model imported from Sonnet's em. 


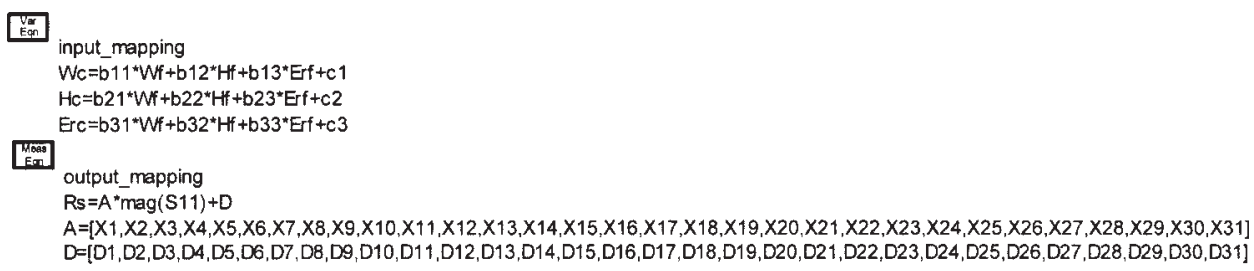

Figure 11. SM surrogate setup in ADS. The VAR component input_mapping deals with $\overline{\boldsymbol{B}}, \overline{\boldsymbol{c}}$. The MeasEqn component output_mapping involves mapping parameters $\bar{A}$ and $\overline{\boldsymbol{d}}$.

$\boldsymbol{R}_{s 2}, \boldsymbol{R}_{s 3}$, and $\boldsymbol{R}_{s 4}$ by fixing mapping parameters $\boldsymbol{B}, \boldsymbol{c}$, $\boldsymbol{A}$, or $\boldsymbol{d}$.

\section{EXAMPLES}

In this section, we illustrate the implementation into the ADS framework of the models introduced in section II. The models are set up using $2 n+1$ base points ( $n$ is the number of design variables), as described in section II. For each example, we perform a statistical analysis of the model error using the set of test points randomly chosen in the region of interest (with uniform distribution). The error is measured as an $l_{2}$ norm of the difference between the fine and the surrogate model responses. It is worth mentioning that we always restrict ourselves to on-grid points in Sonnet's $\boldsymbol{e m}$ for the base and test sets.

\section{A. Microstrip Right-Angle Bend [9]}

The design parameters of the microstrip right-angle bend [5] are width $W$, substrate height $H$, and dielectric constant $\varepsilon_{r}$ [see Figure 12(a)]. The region of interest is $20 \mathrm{mil} \leq W \leq 30 \mathrm{mil}, 8 \mathrm{mil} \leq H \leq 16 \mathrm{mil}$, and $8 \leq \varepsilon_{r} \leq 10$. The frequency range is 1 to $31 \mathrm{GHz}$ in steps of $2 \mathrm{GHz}$ (16 points). The number of base points in the region of interest is 7 . The reference point is $\boldsymbol{x}^{0}=\left[\begin{array}{ll}25 & 12\end{array}\right]^{T}$, the region size $\boldsymbol{\delta}=\left[\begin{array}{lll}5 & 4 & 1\end{array}\right]^{T}$.

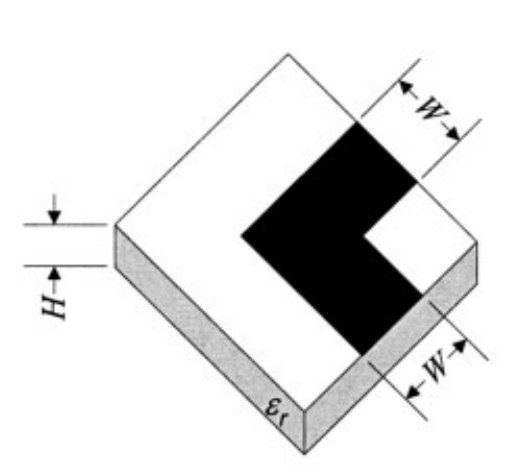

(a)
We develop an Agilent ADS empirical model for the microstrip right-angle bend in Figure 12(a). The fine model is analyzed by Sonnet's $\boldsymbol{e m}$ using $1 \times 1$ mil mesh size and the circuit model is the LC circuit shown in Figure 12(b). The equivalent circuit parameters are calculated from expressions developed by Kirschning, Jansen, and Koster according to their formula in [13].

Table II shows the statistical analysis of the modeling error for 10 random test points. Figures 13 and 14 show error plots for the coarse model $\mathbf{R}_{c}$ and surrogate model $\boldsymbol{R}_{s 4}$, respectively. Figure 15 shows $\left|S_{11}\right|$ versus frequency for the fine model $(-)$, the coarse model $(\times)$, and the $\boldsymbol{R}_{s 2}$ model $(\bigcirc)$ for one test point.

\section{B. Microstrip Shaped T-Junction [9]}

In this example we consider a shaped T-junction [5], as shown in Figure 16(a). The T-junction is symmetric in the sense that all input lines have the same width $w$. The fine model is analyzed by Sonnet's $\mathbf{e m}$ and the coarse model is composed of empirical models of simple microstrip elements [see Figure 16(b)] of Agilent ADS. The fine and coarse model parameters are given by $\boldsymbol{x}_{f}=\left[\begin{array}{lllllll}w & w_{2} & w_{1} & y & x & h & \varepsilon_{r}\end{array}\right]^{T}$ and $\boldsymbol{x}_{c}=\left[\begin{array}{lll}w_{c} & w_{c 2}\end{array}\right.$ $\left.w_{c 1} y_{c} x_{c} h_{c} \varepsilon_{r c}\right]^{T}$. The region of interest is $15 \mathrm{mil} \leq$ $h \leq 25$ mil, 5 mil $\leq x \leq 15$ mil, 5 mil $\leq y \leq 15$ mil, and $8 \leq \varepsilon_{r} \leq 10$. The frequency range used is 2 to 20

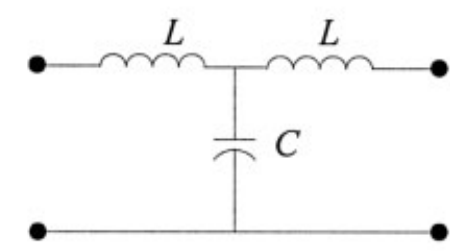

(b)

Figure 12. Microstrip right-angle bend [9]: (a) the fine model; (b) the circuit model. 
TABLE II. Error Statistics for Microstrip Right-Angle Bend Using 10 Random Test Points in the Region of Interest

\begin{tabular}{lcc}
\hline Model & Mean Error & Maximum Error \\
\hline $\boldsymbol{R}_{c}$ & 0.345 & 0.428 \\
$\boldsymbol{R}_{s 1}$ & 0.022 & 0.030 \\
$\boldsymbol{R}_{s 2}$ & 0.012 & 0.030 \\
$\boldsymbol{R}_{s 3}$ & 0.011 & 0.027 \\
$\boldsymbol{R}_{s 4}$ & 0.011 & 0.029 \\
\hline
\end{tabular}

$\mathrm{GHz}$ in steps of $2 \mathrm{GHz}$ (10 points). The width $w$ of the input lines is determined in terms of $h$ and $\varepsilon_{r}$ so that the characteristic impedance of the input lines is $50 \Omega$. The width $w_{1}$ is taken as one-third of the width $w$. The width $w_{2}$ is obtained so that the characteristic impedance of the microstrip line after the step connected to port 2 is twice the characteristic impedance of the microstrip line after the step connected to port 1 [see Figure 16(b)]. The number of base points in the region of interest is nine, since we have only four independent variables $\left\{h, x, y, \varepsilon_{r}\right\}$. The reference point is $\boldsymbol{x}^{0}=$ $\left[\begin{array}{lllllll}21 & 33 & 7 & 10 & 10 & 20 & 9\end{array}\right]^{T}$.

Table III shows the statistical analysis of the modeling error for 24 random test points. Figures 17 and 18 show error plots for the coarse model $\boldsymbol{R}_{c}$ and surrogate model $\boldsymbol{R}_{s 4}$, respectively. Figure 19 shows $\left|S_{11}\right|$ and $\left|S_{22}\right|$ versus frequency for the fine model ( - ), the coarse model $(\times)$, and the $\boldsymbol{R}_{s 2}$ model $(\bigcirc)$ at one test point.

\section{Three-Section Microstrip Transformer}

Next we illustrate the ADS modeling implementation in a detailed three-section microstrip impedance trans-

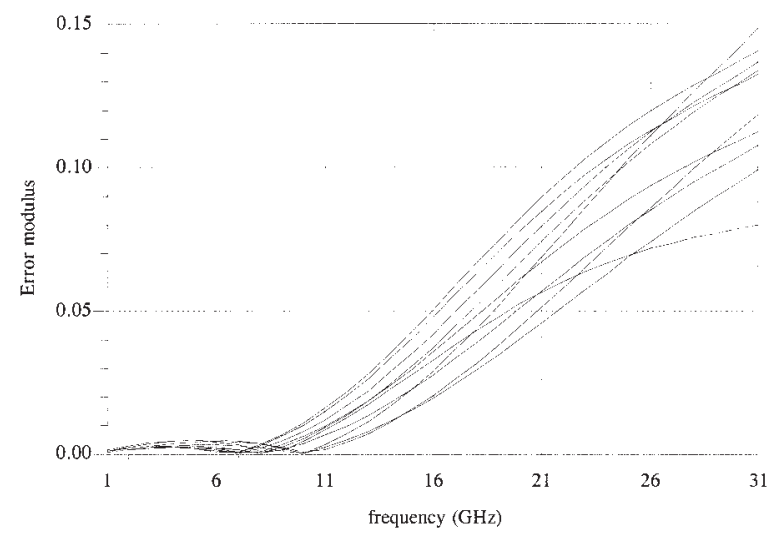

Figure 13. ADS error plots for the $\boldsymbol{R}_{c}$ model (modulus of difference between $\boldsymbol{R}_{f}$ and $\boldsymbol{R}_{c}$ ) for the microstrip right-angle bend (10 test points).

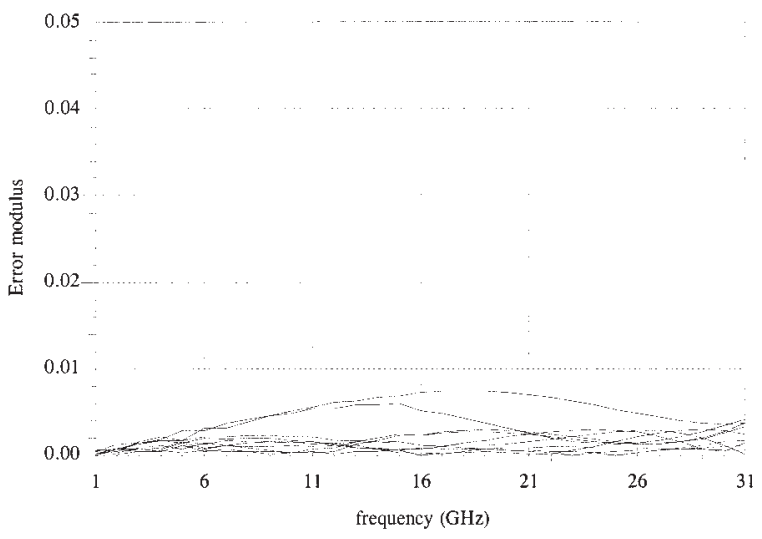

Figure 14. ADS error plots for the $\boldsymbol{R}_{s 4}$ model (modulus of difference between $\boldsymbol{R}_{f}$ and $\boldsymbol{R}_{s 4}$ ) for the microstrip rightangle bend (10 test points).

former [Figure 20(a)] [11]. The fine model is a Sonnet em model. The coarse model is shown in Figure 20(b). It comprises cascaded ADS microstrip empirical models. The fine and coarse model parameters are given by $\boldsymbol{x}_{f}=\left[W_{1} L_{1} W_{2} L_{2} W_{3} L_{3}\right]^{T}$ and $\boldsymbol{x}_{c}=\left[W_{1 c} L_{1 c}\right.$ $\left.W_{2 c} L_{2 c} W_{3 c} L_{3 c}\right]^{T}$. The region of interest is $12.7 \mathrm{mil} \leq$ $W_{1} \leq 15.5 \mathrm{mil}, 105 \mathrm{mil} \leq L_{1} \leq 129 \mathrm{mil}, 5 \mathrm{mil} \leq$ $W_{2} \leq 6.2$ mil, 108 mil $\leq L_{2} \leq 132 \mathrm{mil}, 1.5 \mathrm{mil} \leq$ $W_{3} \leq 1.9 \mathrm{mil}$, and $109 \mathrm{mil} \leq L_{3} \leq 133 \mathrm{mil}$. The frequency range used is 5 to $15 \mathrm{GHz}$ in steps of $1 \mathrm{GHz}$ (11 points). The reflection coefficient $S_{11}$ is used to match the responses of SM-based model and fine model. The number of base points in the region of interest is 13 , since we have only six independent variables $\left\{W_{1}, L_{1}, W_{2}, L_{2}, W_{3}, L_{3}\right\}$. The reference point is $\boldsymbol{x}^{0}=\left[\begin{array}{llllll}14.1 & 117 & 5.6 & 120 & 1.7 & 121\end{array}\right]^{T}$. The thickness of the dielectric substrate is $0.635 \mathrm{~mm}(25$ mil) and its relative permittivity is 9.7 .

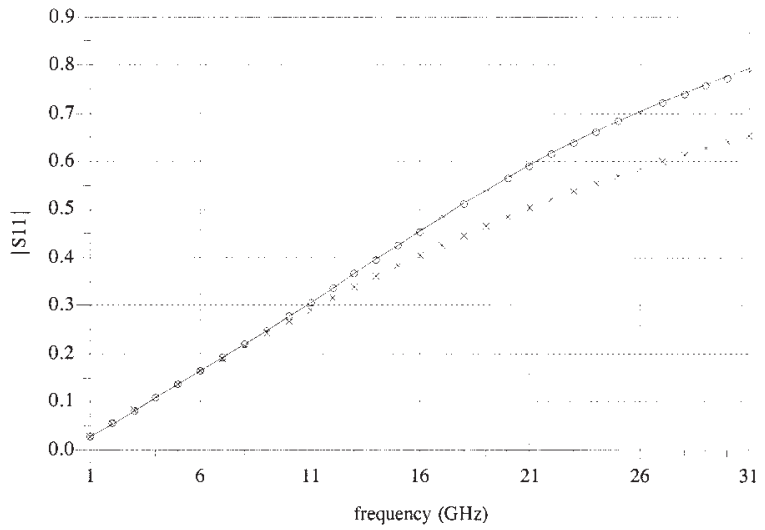

Figure 15. ADS plot of $\left|S_{11}\right|$ for the microstrip right-angle bend: the fine model ( - ), the coarse model $(\times)$, and the $\boldsymbol{R}_{s 2}$ model $(\bigcirc)$ at a test point. 


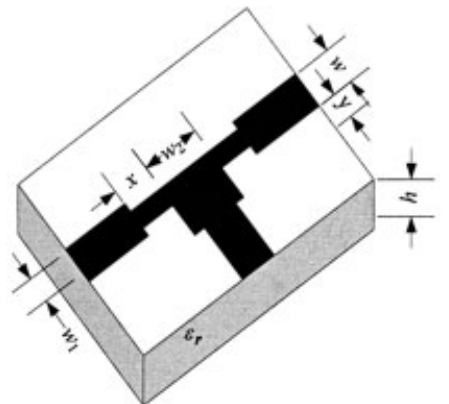

(a)

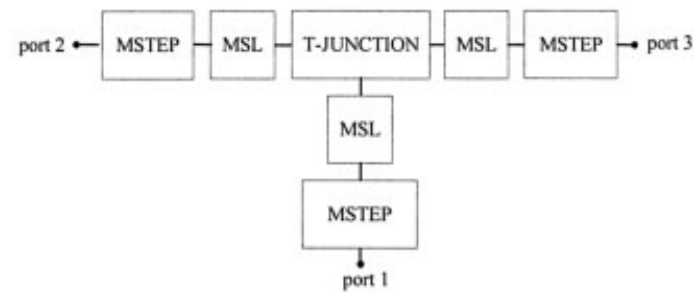

(b)

Figure 16. Microstrip shaped T-junction [9]: (a) the physical structure (fine model); (b) the coarse model.

The fine model is imported using the S2PMDIF component (Figure 21). Prior to ADS simulation (modeling), we generated 13 base and 20 test Sonnet's $\boldsymbol{e m}$.son files using an internal Matlab geometry capture [14] program. The 33 files were simulated in Sonnet's $\mathbf{e m}$ batch mode to produce 33 individual output (response) MDF files instead of one large MDF file. This is slightly different from the implementations in Figure 10 and subsections A and B. During

TABLE III. Error Statistics for Microstrip Shaped T-Junction Using 24 Random Test Points in the Region of Interest

\begin{tabular}{lcc}
\hline Model & Mean Error & Maximum Error \\
\hline $\boldsymbol{R}_{c}$ & 0.1481 & 0.207 \\
$\boldsymbol{R}_{s 1}$ & 0.0075 & 0.014 \\
$\boldsymbol{R}_{s 2}$ & 0.0062 & 0.013 \\
$\boldsymbol{R}_{s 3}$ & 0.0053 & 0.011 \\
$\boldsymbol{R}_{s 4}$ & 0.0052 & 0.010 \\
\hline
\end{tabular}

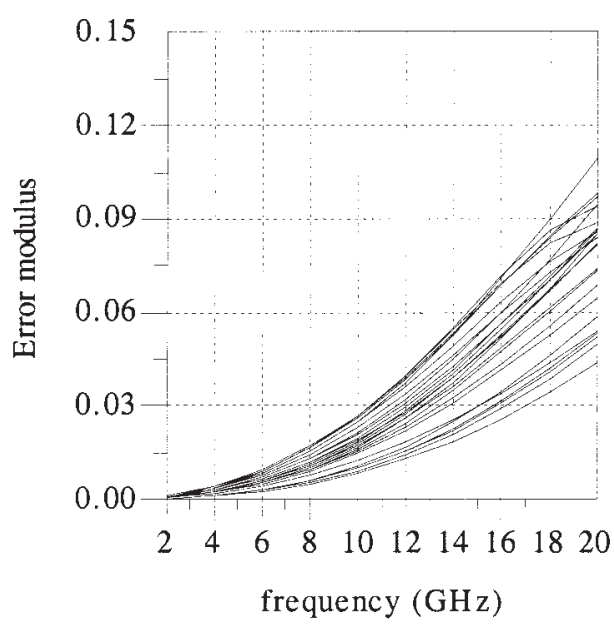

(a)
ADS simulation, the output filename and parameter list files (Figure 22) are imported and swept using the DAC and parameter sweep, as shown in Figure 23. The goal and optimization algorithm components for the modeling are shown in Figure 24. Figure 25 shows the design parameters, input mapping, and parameter initial values. To avoid tedious typing, a simple Matlab program is written to generate the input mapping matrix multiplication data block. The whole data block can be copied and pasted into the VAR component using a "Name = Value" entry mode. The same procedure is used for generating the starting mapping parameter value block for the output mapping (see Figure 26).

Table IV shows the statistical analysis of the modeling error for 20 random test points. Figure 27 shows the error plots for the coarse model $\boldsymbol{R}_{c}$ and surrogate model $\boldsymbol{R}_{s 4}$, respectively.

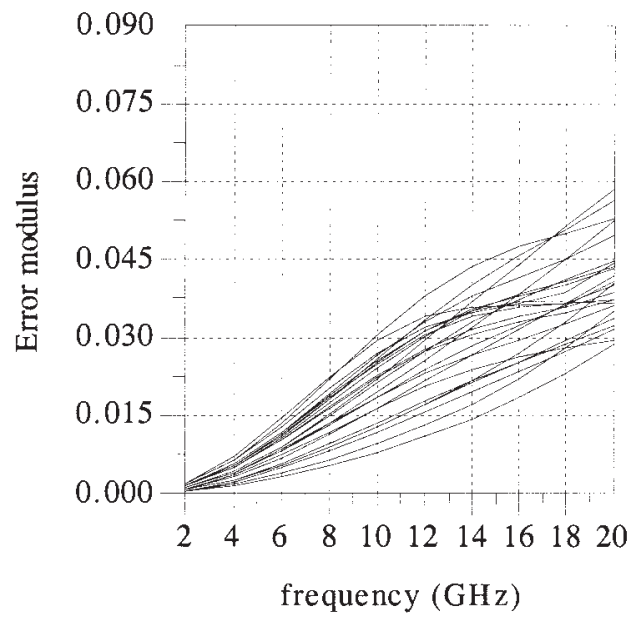

(b)

Figure 17. ADS error plots for the $\boldsymbol{R}_{c}$ model (modulus of difference between $\boldsymbol{R}_{f}$ and $\boldsymbol{R}_{c}$ ) for the microstrip shaped T-junction (24 test points): (a) $\left|S_{11}\right|$; (b) $\left|S_{22}\right|$. 


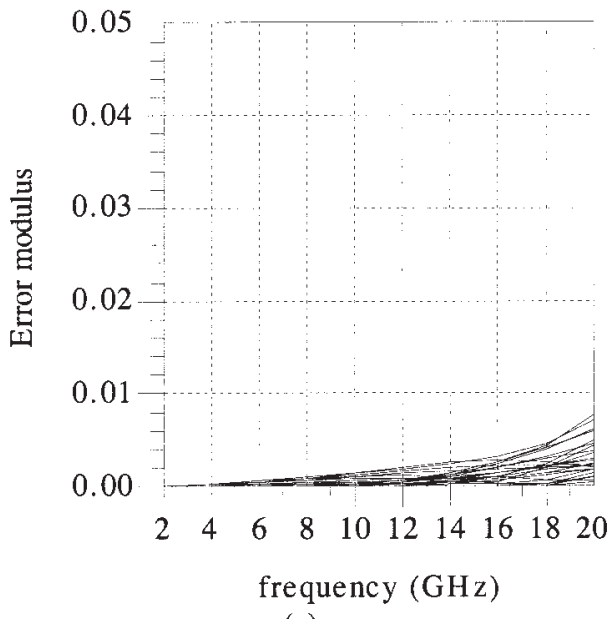

(a)

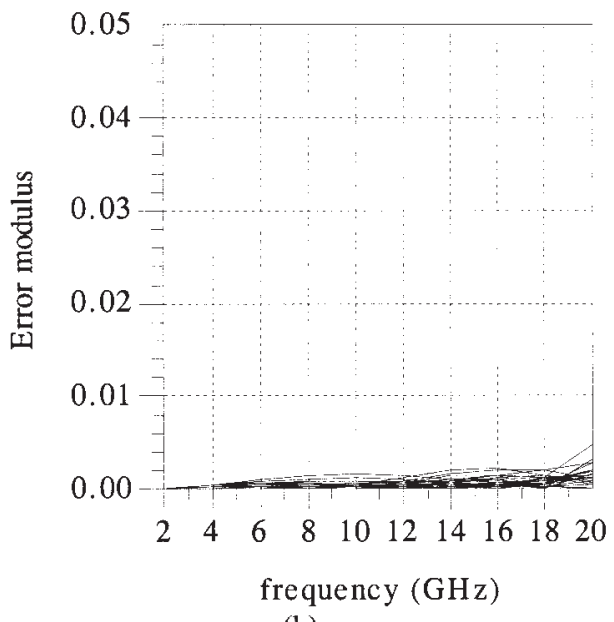

(b)

Figure 18. ADS error plots for the $\boldsymbol{R}_{s 4}$ model (modulus of difference between $\boldsymbol{R}_{f}$ and $\boldsymbol{R}_{s 4}$ ) for the microstrip shaped T-junction (24 test points): (a) $\left|S_{11}\right|$; (b) $\left|S_{22}\right|$.

Now we can start to use the model we obtained from the modeling procedure. A few changes need to be made to the schematic. Since the modeling uses a fixed frequency sweep, the sizes of matrix $\boldsymbol{A}$ and vector $\boldsymbol{d}$ are not flexible for any other desired frequency sweep. We use two ways to interpolate the frequency sweep according to section II: (i) we interpolate $\boldsymbol{R}_{s}$ directly using the Eqn component in the data display window (see Figure 28), and the interp function generates interpolated responses between $m$ frequency points; or (ii) as in Figure 29, the optimized matrix $\boldsymbol{A}$ and vector $\boldsymbol{d}$ are saved in files and imported using the DAC with linear interpolation (Figure 30). In either way, the model is ready for any frequency sweep. From Figure 31, we can see that interpolating $\boldsymbol{A}$ and $\boldsymbol{d}$ gives a smoother curve than interpolating $\boldsymbol{R}_{\boldsymbol{s}}$ directly. Setting the design parameters to be optimizable, we can optimize the new model by changing the goal and optimization algorithm to a minimax design, as shown in Figure 32. We can also do yield optimization by simply changing the setting (Figure 33). We show a yield-analysis responses plot and histogram in Figures 34 and 35, respectively. When using a 5\% tolerance, $63 \%$ yield is obtained using the ADS SM-based model. We apply yield analysis to the Sonnet's em fine model using the same specification and obtain $56 \%$ yield (Figures 36 and 37). The discrepancy between

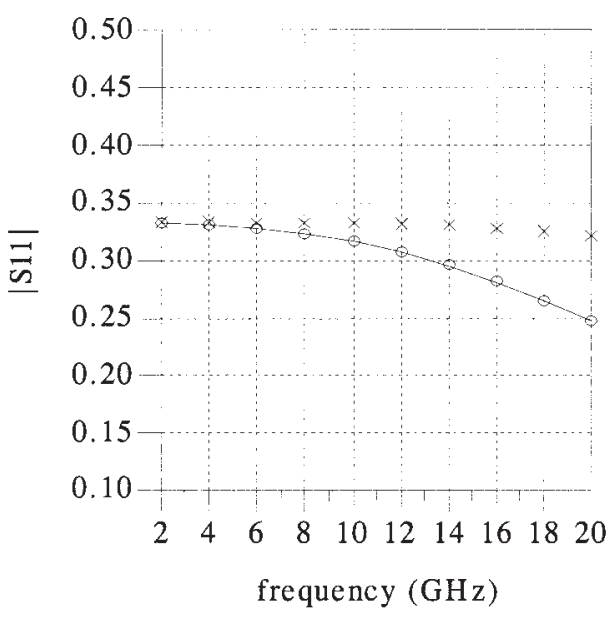

(a)

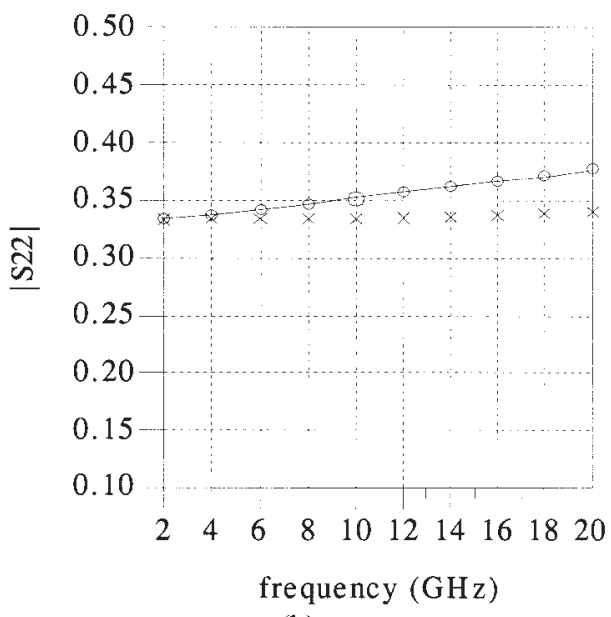

(b)

Figure 19. ADS plot of $\left|S_{11}\right|$ and $\left|S_{22}\right|$ for the microstrip shaped T-junction: the fine model (-), the coarse model $(\times)$, and the $\boldsymbol{R}_{s 2}$ model $(\bigcirc)$ at a test point. 


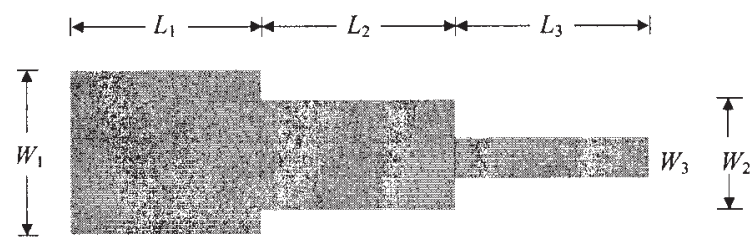

(a)

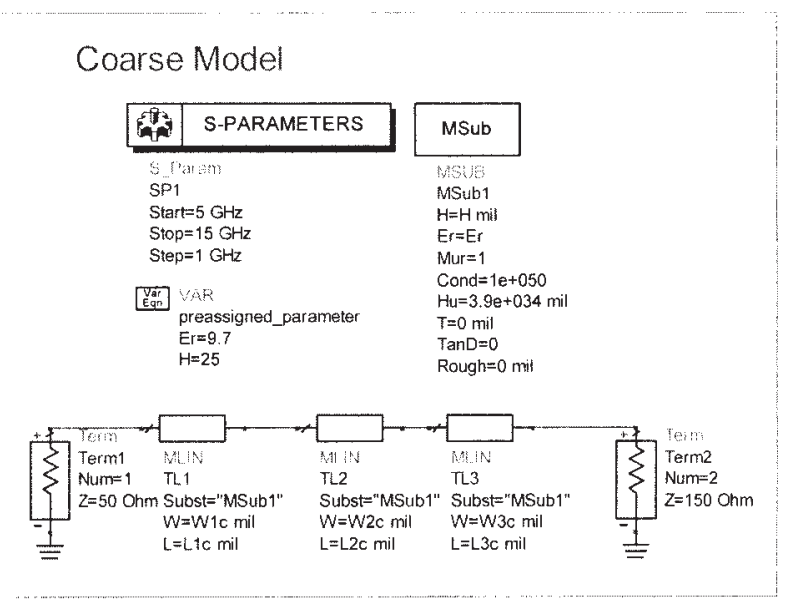

(b)

Figure 20. The three-section 3:1 microstrip impedance transformer [11]: (a) structure and dimensions; (b) ADS coarse model.

the fine model and the SM-based ADS model can be explained as follows: (a) the SM-based model is not perfect; (b) the number of random samples is relatively small for statistical analysis; (c) the fine model is snapped to the grid. The 5\% variation in

\section{Fine Model}

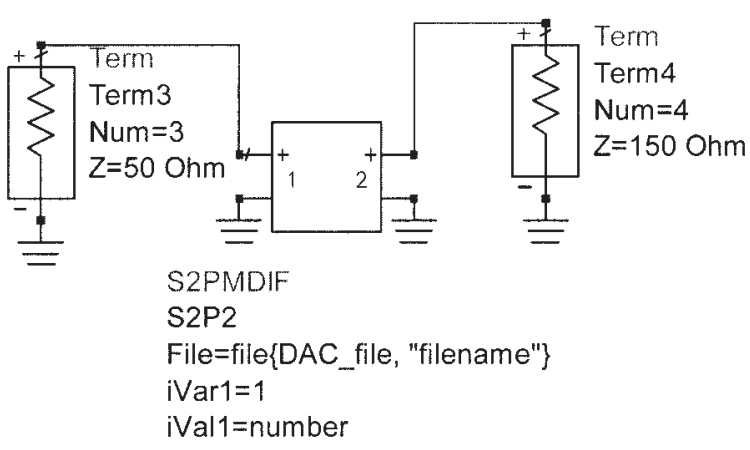

Figure 21. Three-section microstrip transformer schematic: S2PMDIF component imports the fine-model responses.

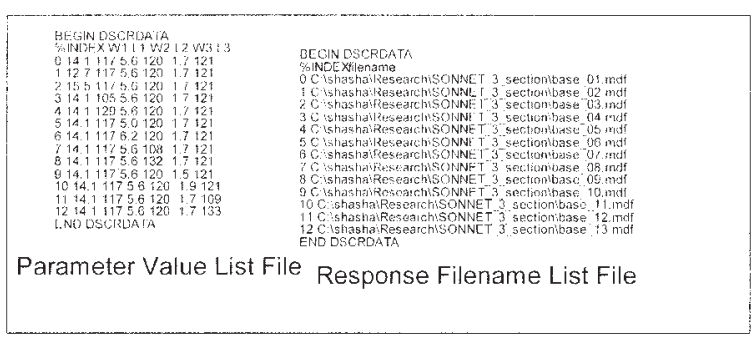

Figure 22. Three-section microstrip transformer schematic: parameter value list and response filename list file. certain parameters, for example, $w_{3}$, is too small to change the structure vertex to the next grid.

\section{HTS Filter Example}

We consider the HTS bandpass filter of [15]. The physical structure is shown in Figure 38(a). The design variables are the lengths of the coupled lines and the separation between them, namely,

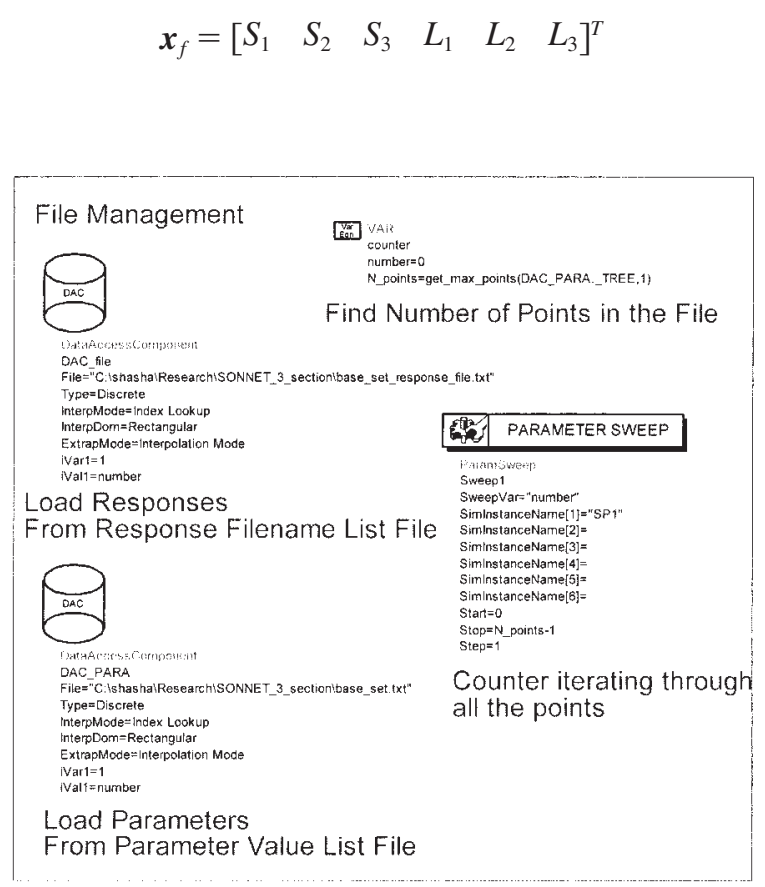

Figure 23. Three-section microstrip transformer schematic: sweep through the parameter values and response files. 


\begin{tabular}{|c|c|c|c|}
\hline GOAL & 5 & OPTIM & \\
\hline 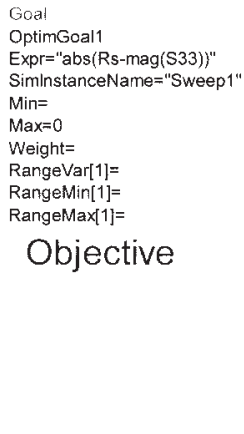 & $\begin{array}{l}\text { Opti } \\
\text { Optit } \\
\text { Opti } \\
\text { Ero } \\
\text { Max } \\
\text { Des } \\
\text { Stat } \\
\text { Fina } \\
\text { Narr } \\
\text { Set } \\
\text { See } \\
\text { Sav } \\
\text { Sav } \\
\text { Sav } \\
\text { Upd } \\
\text { Sav } \\
\text { Sav }\end{array}$ & 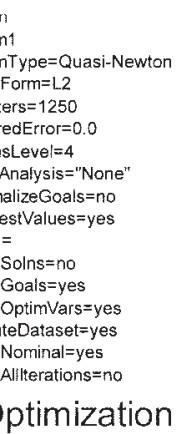 & $\begin{array}{l}\text { UseAilloptVars =yes } \\
\text { UseAllloals=yes } \\
\text { SaveCUurentEF }=\text { no }\end{array}$ \\
\hline
\end{tabular}

Figure 24. Three-section microstrip transformer schematic: optimization goal and algorithm.

The substrate used is lanthanum aluminate with $\varepsilon_{r}=$ 23.425, $H=20 \mathrm{mil}$, and substrate dielectric loss tangent of 0.00003 . The length of the input and output lines is $L_{0}=50 \mathrm{mil}$ and the lines are of width $W=7$ mil.

We use an Agilent ADS coarse model as in [16], which consists of empirical models for single and coupled microstrip transmission lines [see Figure 38(b)]. Notice the symmetry in the HTS structure, that is, coupled lines "CLin5" are identical to "CLin1" and "CLin4" to "CLin2". The fine and coarse model parameters are given by $\boldsymbol{x}_{f}=\left[S_{1} S_{2} S_{3} L_{1} L_{2} L_{3}\right]^{T}$ and $\boldsymbol{x}_{c}$ $=\left[\begin{array}{llllll}S_{1 c} & S_{2 c} & S_{3 c} & L_{1 c} & L_{2 c} & L_{3 c}\end{array}\right]^{T}$.

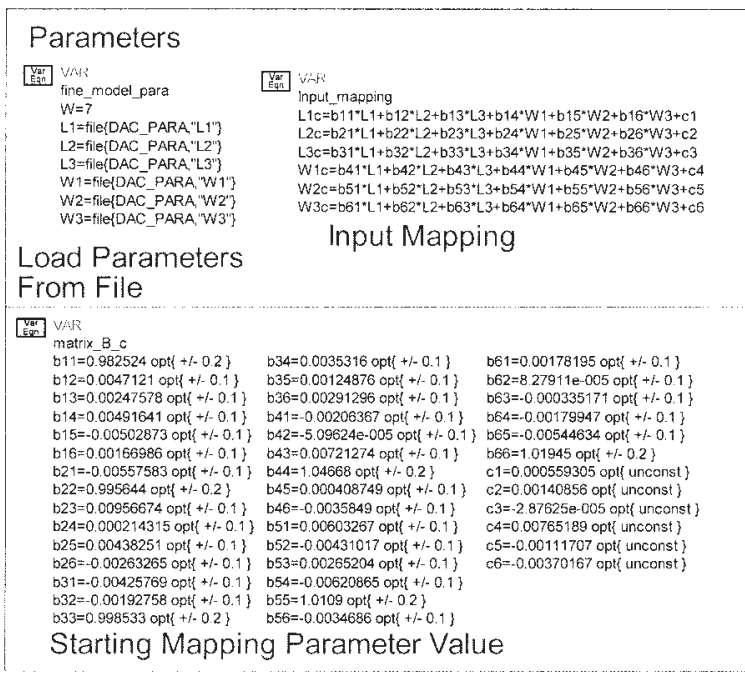

Figure 25. Three-section microstrip transformer schematic: design parameters, input mapping and starting mapping parameter values.

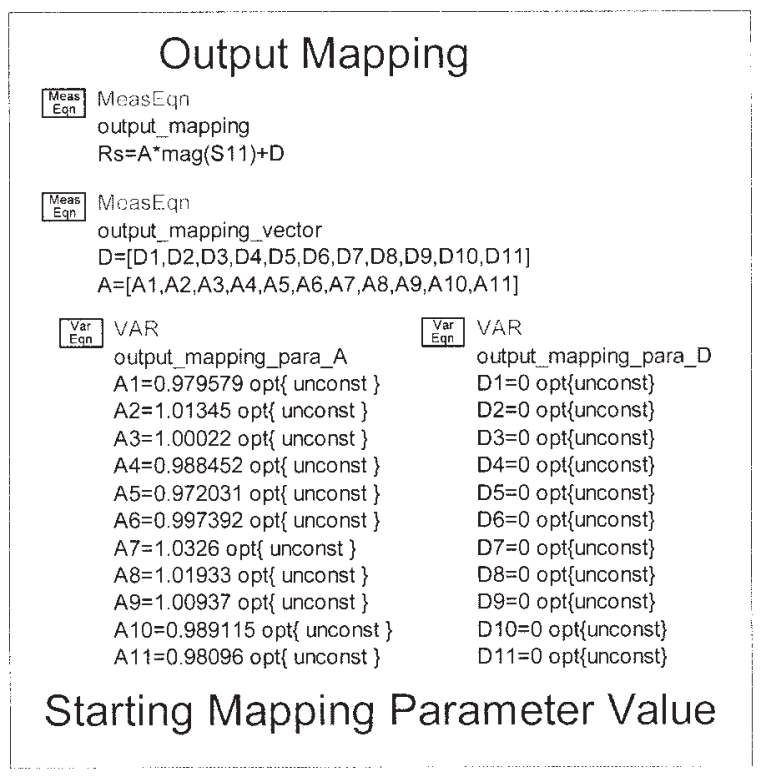

Figure 26. Three-section microstrip transformer schematic: output mapping and starting mapping parameter values.

The fine model is simulated by Sonnet's em using a $1 \times 1$ mil mesh size. Table $\mathrm{V}$ shows the region of interest [6]. The statistical analysis of the modeling error for 20 random test points is given in Table VI. Figures 39(a) and 39(b) show error plots for the coarse model $\boldsymbol{R}_{c}$ and surrogate model $\boldsymbol{R}_{s 4}$, respectively. Figure 40 shows $\left|S_{21}\right|$ versus frequency for the fine model ( - ), the coarse model $(\times)$, and the $R_{s 4}$ model $(\bigcirc)$ at one test point. Figure 41 shows an $\left|S_{21}\right|$ comparison for the HTS filter at three test points, where the $\boldsymbol{R}_{s 4}$ model is interpolated and finely swept. The results are similar to those obtained by frequency partial-space-mapped neuromodeling [6].

\section{DISCUSSION AND FURTHER IMPROVEMENT OF SM MODELING}

The modeling methodology presented in this article is simple and has the important advantage of requiring a

TABLE IV. Error Statistics for Three-Section Microstrip Transformer Using 20 Random Test Points in the Region of Interest

\begin{tabular}{lcc}
\hline Model & Mean Error & Maximum Error \\
\hline $\boldsymbol{R}_{c}$ & 0.042 & 0.057 \\
$\boldsymbol{R}_{s 1}$ & 0.009 & 0.015 \\
$\boldsymbol{R}_{s 2}$ & 0.008 & 0.016 \\
$\boldsymbol{R}_{s 3}$ & 0.007 & 0.014 \\
$\boldsymbol{R}_{s 4}$ & 0.006 & 0.012 \\
\hline
\end{tabular}




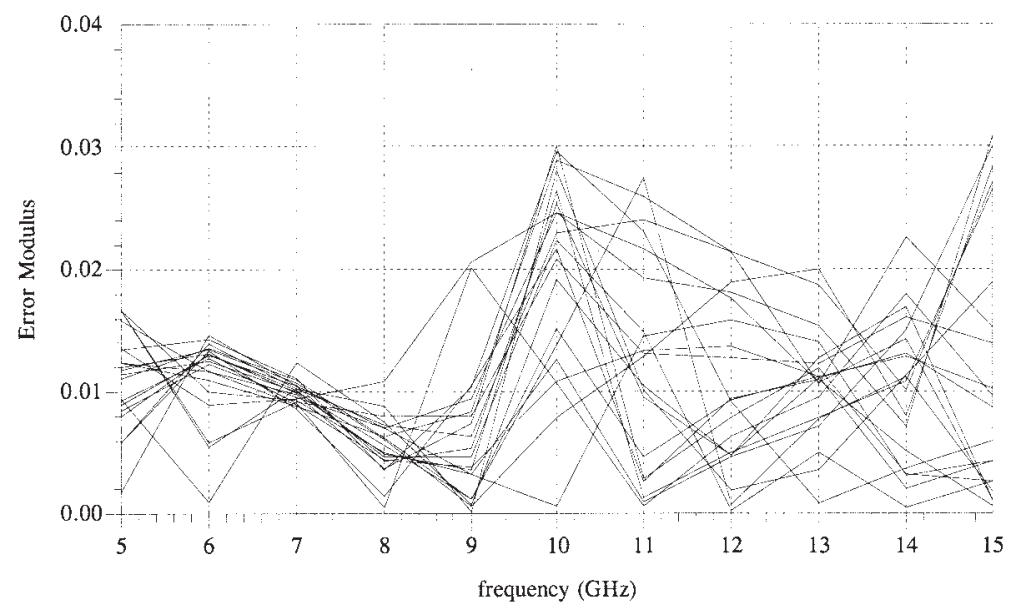

(a)

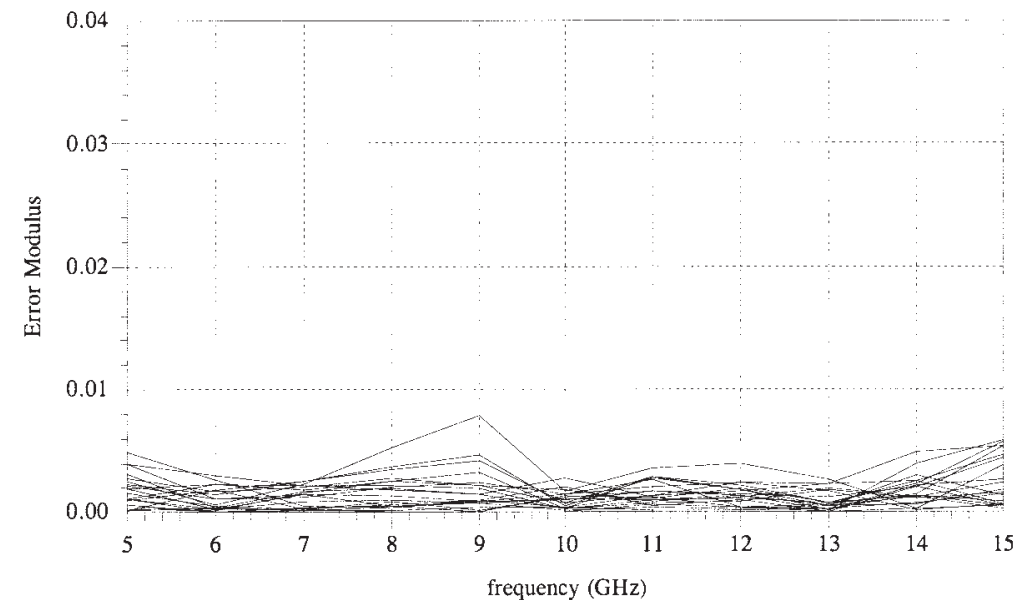

(b)

Figure 27. ADS error plots for the three-section microstrip transformer (20 test points)

\section{Eqn Rsi=interp(SP2.Rs,5GHz, 15Ghz,0.1Ghz)}

Figure 28. Three-section microstrip transformer schematic: direct interpolation of the responses.

\begin{tabular}{|c|c|}
\hline 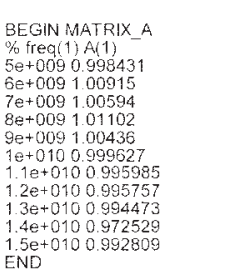 & $\begin{array}{l}\text { BEGIN VECTOR.D } \\
\text { \% Freq(1) dif } 1 \text {. } \\
5 e+009-0.000486849 \\
6 e+009-0.00110578 \\
7 e+000-0.00182379 \\
8 e+009-0.00330207 \\
9 e+009-0.00313116 \\
1 e+010-0.000371335 \\
1.1 e+0100.00271826 \\
12 e+0100.00223473 \\
1.3 e+0100.00119748 \\
14 e+0100.000750631 \\
1.5 e+010-0.00170169 \\
\text { END }\end{array}$ \\
\hline
\end{tabular}

Figure 29. Three-section microstrip transformer schematic: output mapping and starting mapping parameter values.

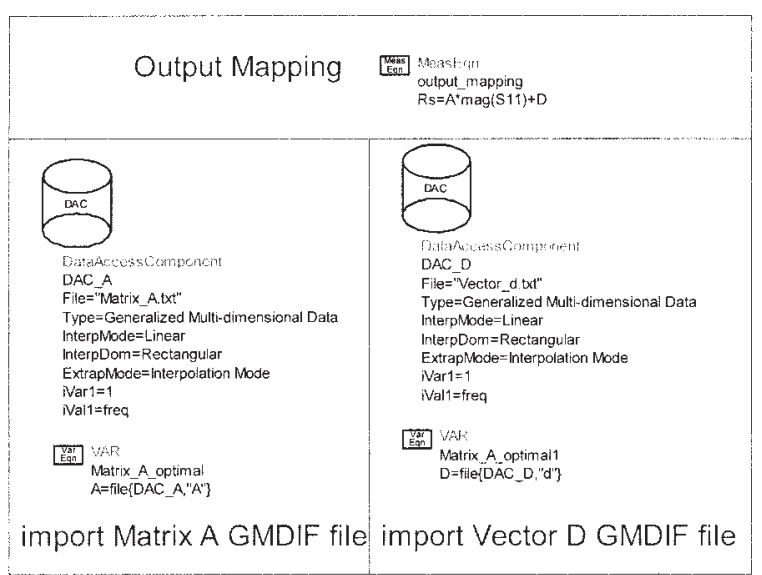

Figure 30. Three-section microstrip transformer schematic: interpolation of the output mapping parameter values. 


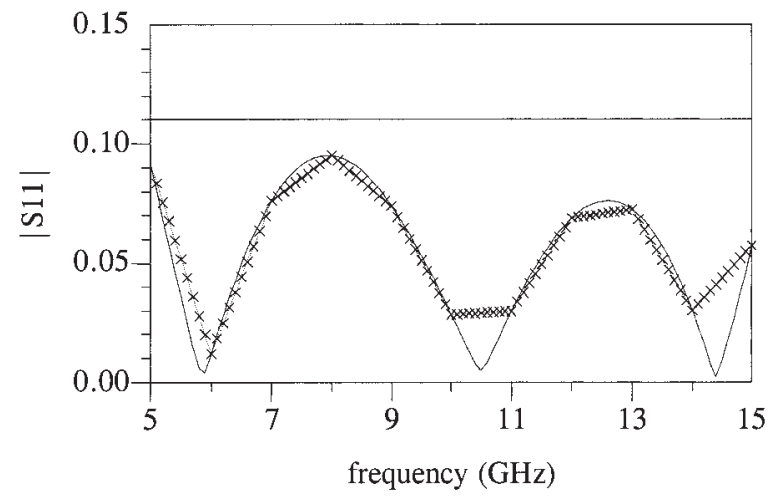

Figure 31. ADS plot for the three-section microstrip transformer: (a) interpolating $\boldsymbol{A}$ and $\boldsymbol{d}(-)$; (b) interpolating $\boldsymbol{R}_{\boldsymbol{s}}$ directly $(\times)$.

small amount of fine-model data. The model is computationally inexpensive, since the only effort is related to the parameter-extraction process while establishing the model - after that, evaluation of the model

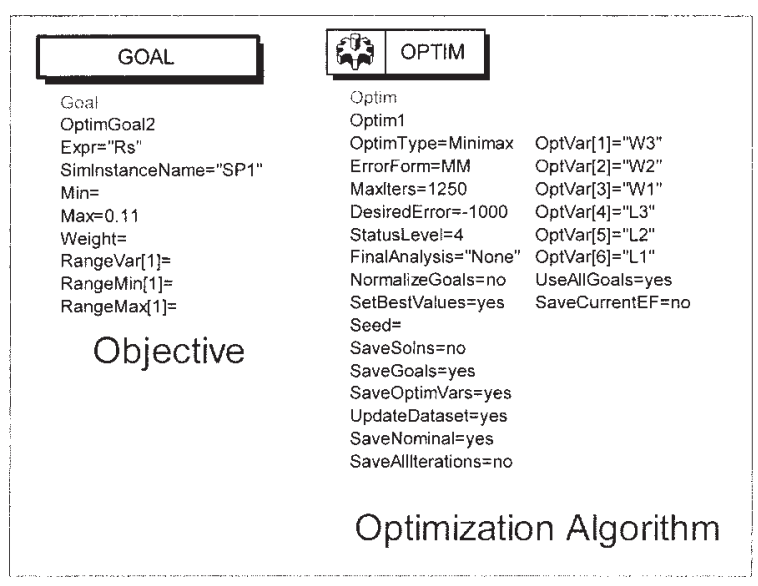

Figure 32. Three-section microstrip transformer schematic: minimax-optimization setting.

\begin{tabular}{|c|c|}
\hline YIELD SPEC & 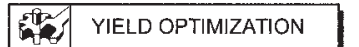 \\
\hline 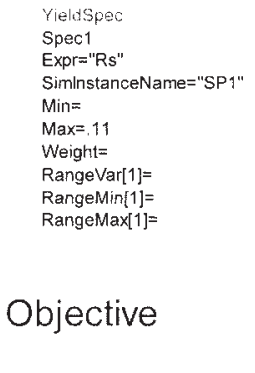 & 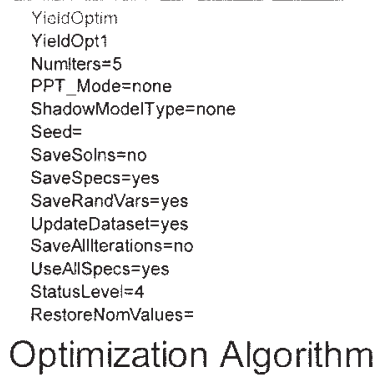 \\
\hline
\end{tabular}

Figure 33. Three-section microstrip transformer schematic: yield-optimization setting.

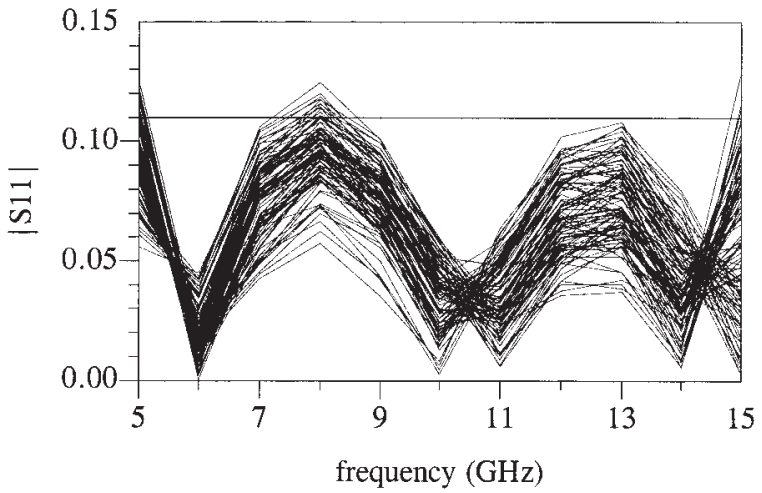

Figure 34. ADS plot for the three-section microstrip transformer: $5 \%$ tolerance yield analysis of 100 points.

is simply the evaluation of suitable formulas with predetermined coefficients. This approach works fine and the performance of the models is as expected; in particular, increasing the number of degrees of freedom usually improves performance (see examples in section IV). Further increasing complexity of the

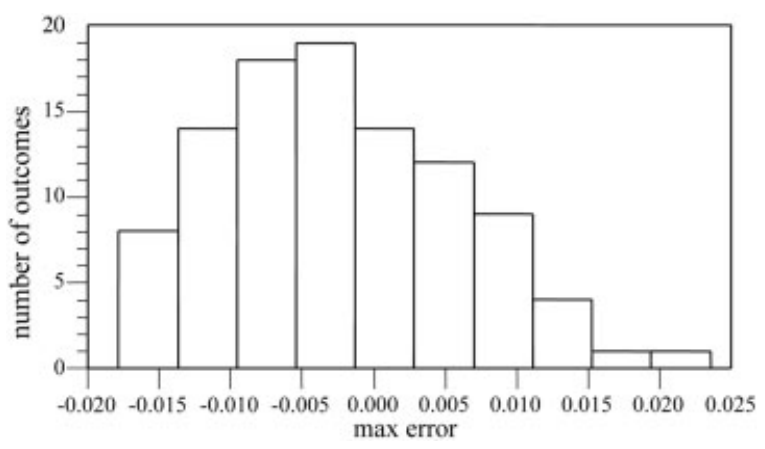

Figure 35. ADS plot for the three-section microstrip transformer 5\% tolerance histogram: $63 \%$ yield of 100 points.

$$
0.15
$$

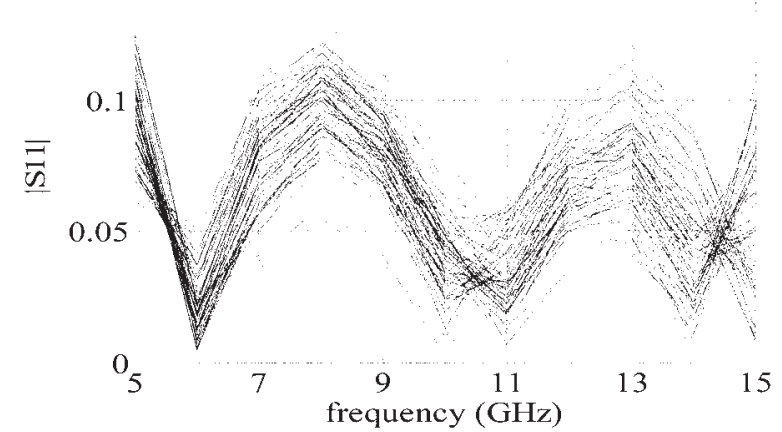

Figure 36. Matlab plot for the three-section microstrip transformer: fine model 100 points yield analysis. 


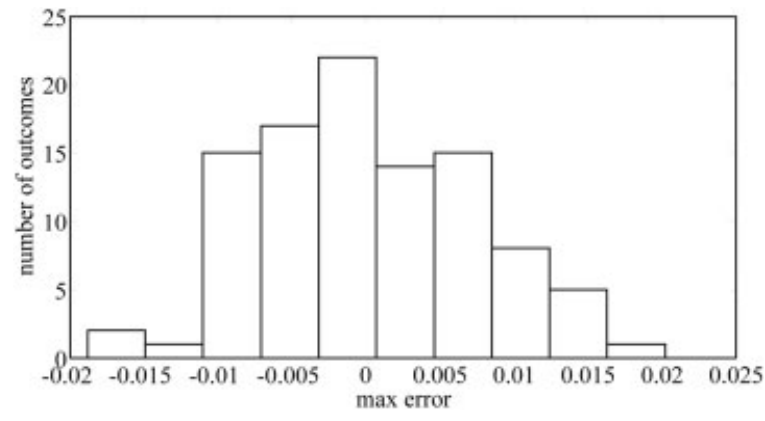

Figure 37. Matlab plot for the three-section microstrip transformer: fine model $56 \%$ yield of 100 points.

model (for example, $\boldsymbol{R}_{s 4}$ versus $\boldsymbol{R}_{s 3}$ ) has little or no impact.

Further improvement of model accuracy requires a larger number of fine-model data in the creation of the model, that is, a larger number of base points (currently $2 n+1$ ). Similarly, flexibility of the surrogates should be improved to follow nonlinearity of the fine model. Possible methods of increasing model flexibility include: dividing the frequency band into subbands (problems: analytical properties of the model suffer, problems with parameter extraction arise-

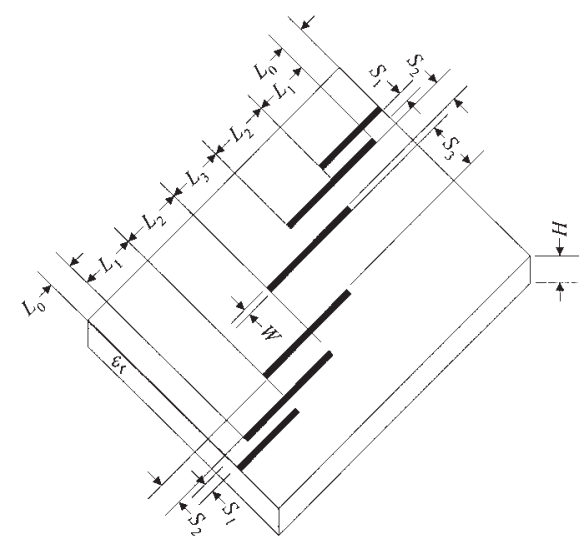

(a)

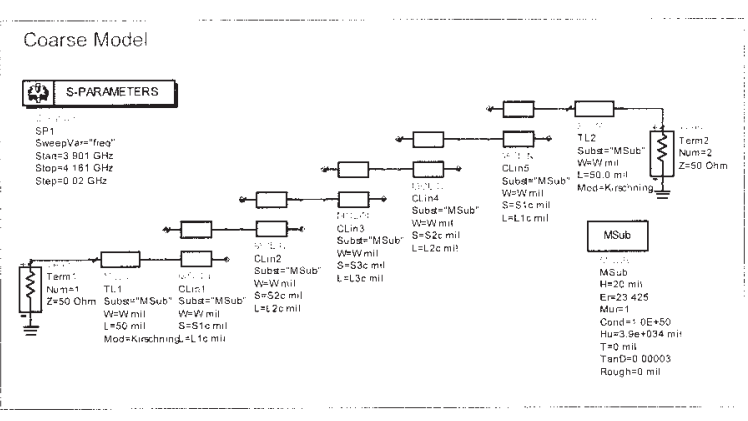

(b)

Figure 38. HTS filter: (a) the physical structure [15]; (b) the coarse model [16] as implemented in Agilent ADS [8].
TABLE V. The Region of Interest

\begin{tabular}{lcc}
\hline Parameter & Reference Point $\left(\boldsymbol{x}^{0}\right)$ & Region Size $(\delta)$ \\
\hline$L_{1}$ & 180 & 5 \\
$L_{2}$ & 200 & 10 \\
$L_{3}$ & 180 & 5 \\
$S_{1}$ & 20 & 2 \\
$S_{2}$ & 80 & 5 \\
$S_{3}$ & 80 & 10 \\
\hline
\end{tabular}

nonuniqueness of solutions, and so on), adding external terms (linear or nonlinear), and hybrid models, for example, SM combined with classical approximation (problems: classical methods are not physics-based and can cause large errors while moving away from the center of the region of interest). These approaches have been partially implemented [17]. The above methods can only help if the low accuracy of the model is caused by limited flexibility of the model. A good indication of that is the parameter-extraction error at the base points.

A small parameter-extraction error at the base points (or, equivalently, good model accuracy at the base points) indicate that the only way of increasing model accuracy is to include more fine-model data. Unfortunately, SM is not suitable for handling a large amount of fine-model data by itself, that is, increasing the number of base points does not help if the number of degrees of freedom of the model remains unchanged. Possible solutions are: (i) dividing the region of interest into smaller subregions so that a separate SM model is set up for each subregion (problems: the number of regions grows exponentially with the dimension of the design parameter space; a common problem with subregions can be discontinuity or at least a lack of higher-order regularity at the border between the regions); (ii) as above, but setting up one SM model for the whole region and individual models for subregions based on classical approximation/interpolation methods.

TABLE VI. Error Statistics for HTS Filter Using 20 Random Test Points in the Region of Interest

\begin{tabular}{lcc}
\hline Model & Mean Error & Maximum Error \\
\hline $\boldsymbol{R}_{c}$ & 1.508 & 2.275 \\
$\boldsymbol{R}_{s 1}$ & 0.158 & 0.259 \\
$\boldsymbol{R}_{s 2}$ & 0.149 & 0.241 \\
$\boldsymbol{R}_{s 3}$ & 0.153 & 0.242 \\
$\boldsymbol{R}_{s 4}$ & 0.152 & 0.235 \\
\hline
\end{tabular}




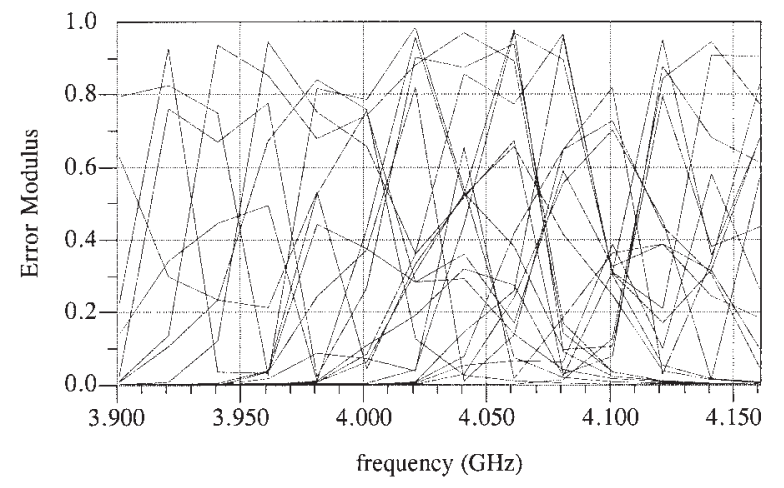

(a)

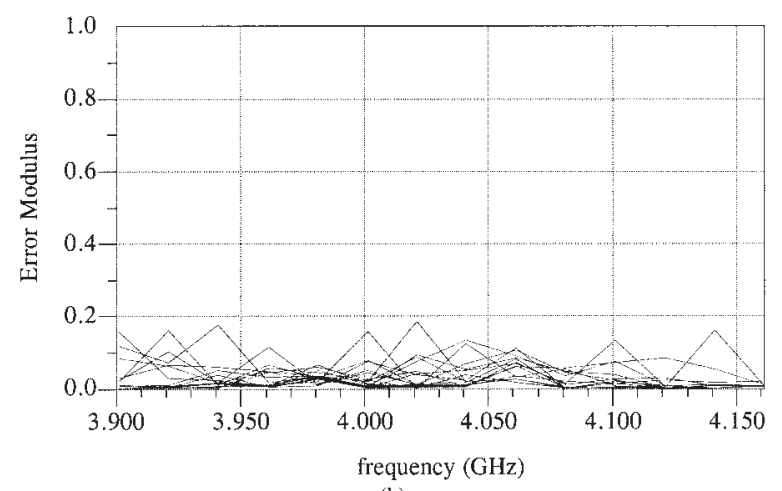

(b)

Figure 39. ADS error plots for the HTS filter (20 test points): (a) the $\boldsymbol{R}_{c}$ model (modulus of difference between $\boldsymbol{R}_{f}$ and $\boldsymbol{R}_{c}$ ); (b) the $\boldsymbol{R}_{s 4}$ model (modulus of difference between $\boldsymbol{R}_{f}$ and $\boldsymbol{R}_{s 4}$ ).

\section{CONCLUSION}

In this article, we have presented a simplified SMbased surrogate methodology for RF and microwave CAD modeling, implemented and verified entirely in Agilent ADS. We show that it is easy to switch between the surrogates in the ADS schematic. The surrogates are easy to use as enhanced library models. The models offer good accuracy that is much better in

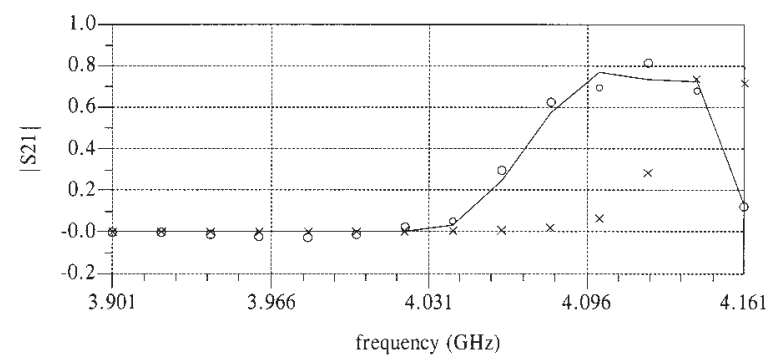

Figure 40. ADS plot of $\left|S_{21}\right|$ for the the HTS filter: the fine model ( - ), the coarse model $(\times)$, and the $\boldsymbol{R}_{s 4}$ model $(\bigcirc)$ at a test point.
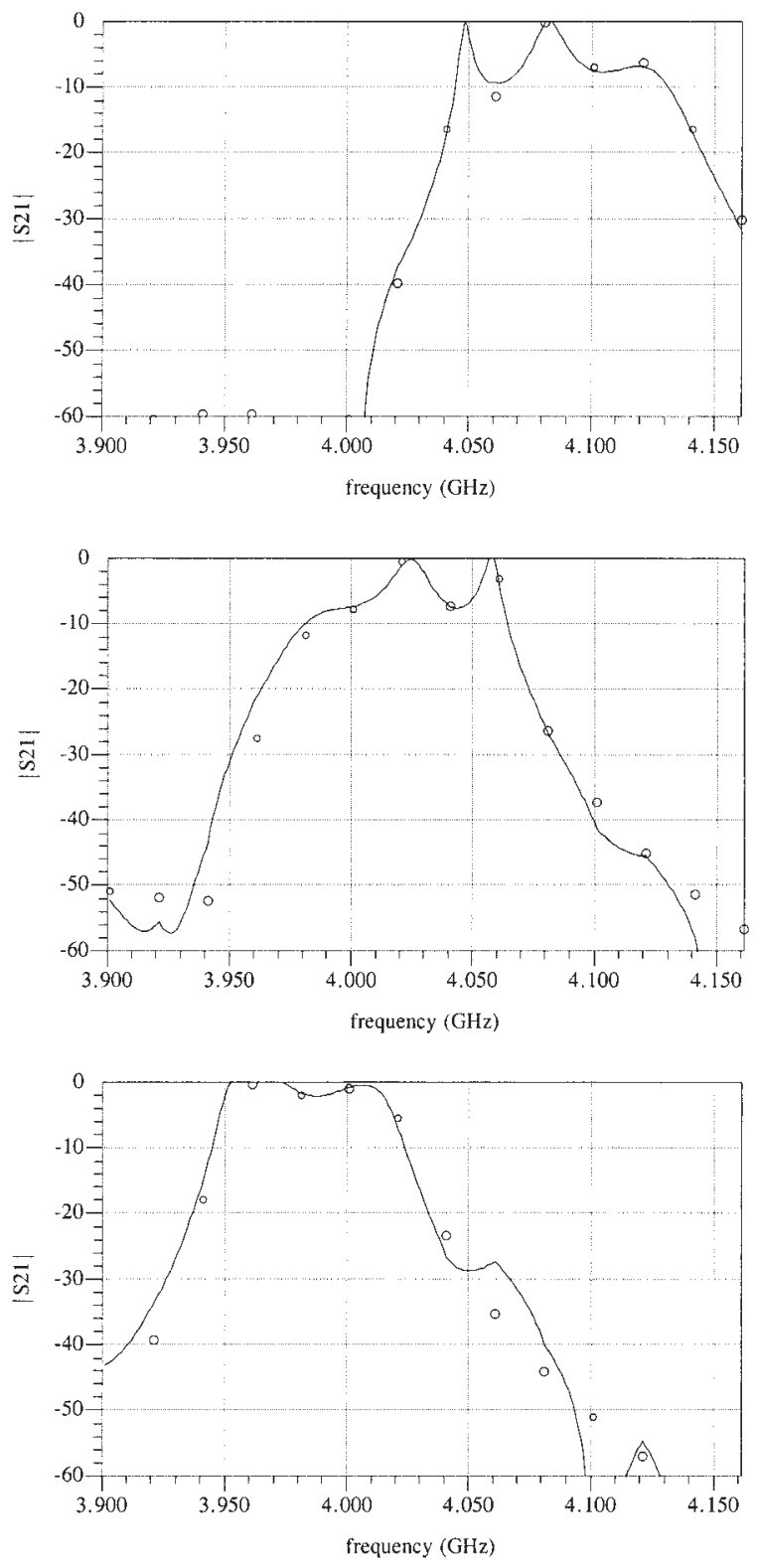

Figure 41. ADS plot of $\left|S_{21}\right|$ for the HTS filter: the fine model (O) and the $\boldsymbol{R}_{s 4}$ model ( - ) at three test points.

our examples than in the models on which they are based.

\section{ACKNOWLEDGMENTS}

We thank Sonnet Software, Inc., Syracuse, NY for $\boldsymbol{e m}^{\mathrm{TM}}$ and Agilent Technologies, Santa Rosa, CA for ADS, as well as for prompt, effective technical support. We thank Dr. M.A. Ismail, Com Dev, Canada for his help in setting up the coarse models, as well as Dr. N.K. Nikolova, McMaster University and Dr. Q.J. Zhang, Carleton University for their help. 


\section{REFERENCES}

1. J.W. Bandler, R.M. Biernacki, S.H. Chen, P.A. Grobelny, and R.H. Hemmers, Space mapping technique for electromagnetic optimization, IEEE Trans Microwave Theory Tech 42 (1994), 2536-2544.

2. J.W. Bandler, R.M. Biernacki, S.H. Chen, R.H. Hemmers, and K. Madsen, Electromagnetic optimization exploiting aggressive space mapping, IEEE Trans Microwave Theory Tech 43 (1995), 2874-2882.

3. J.W. Bandler, Q.S. Cheng, S.A. Dakroury, A.S. Mohamed, M.H. Bakr, K. Madsen, and J. Søndergaard, Space mapping: The state of the art, IEEE Trans Microwave Theory Tech 52 (2004), 337-361.

4. M.H. Bakr, J.W. Bandler, and N. Georgieva, Modeling of microwave circuits exploiting space derivative mapping, 1999 IEEE MTT-S Int Microwave Symp Dig, Anaheim, CA, June 1999, pp. 715-718.

5. J.W. Bandler, N. Georgieva, M.A. Ismail, J.E. RayasSánchez, and Q.J. Zhang, A generalized space mapping tableau approach to device modeling, IEEE Trans Microwave Theory Tech 49 (2001), 67-79.

6. J.W. Bandler, M.A. Ismail, J.E. Rayas-Sánchez, and Q.J. Zhang, Neuromodeling of microwave circuits exploiting space mapping technology, IEEE Trans Microwave Theory Tech 47 (1999), 2417-2427.

7. L. Zhang, J.J. Xu, M. Yagoub, R.T. Ding, and Q.J. Zhang, Efficient analytical formulation and sensitivity analysis of neuro-space mapping for nonlinear microwave device modeling, IEEE Trans Microwave Theory Tech 53 (2005), 2752-2767.

8. Agilent ADS, Version 2003A, Agilent Technologies, 1400 Fountaingrove Parkway, Santa Rosa, CA, 2003.

9. J.W. Bandler, Q.S. Cheng, and S. Koziel, Implementable space mapping approach to enhancement of mi- crowave device models, 2005 IEEE MTT-S Int Microwave Symp, Long Beach, CA, 2005, 1139-1142.

10. S. Koziel, J.W. Bandler, and K. Madsen, Towards a rigorous formulation of the space mapping technique for engineering design, Int Symp Circ Syst (ISCAS), Kobe, Japan, 2005, pp. 5605-5608.

11. J.W. Bandler, Q.S. Cheng, D.M. Hailu, and N.K. Nikolova, A space mapping design framework, IEEE Trans Microwave Theory Tech 52 (2004), 2601-2610.

12. $\boldsymbol{e m}^{\mathrm{TM}}$ Version 9.52, Sonnet Software, Inc., North Syracuse, NY.

13. M. Kirschning, R.H. Jansen, and N.H.L. Koster, Measurement and computer-aided modeling of microstrip discontinuities by an improved resonator method, 1983 IEEE MTT-S Int Microwave Symp, Boston, MA, 1983, pp. 495-497.

14. J.W. Bandler, R.M. Biernacki, and S.H. Chen, Parameterization of arbitrary geometrical structures for automated electromagnetic optimization, Int J RF and Microwave CAE 9 (1999), 73-85.

15. J.W. Bandler, R.M. Biernacki, S.H. Chen, W.J. Getsinger, P.A. Grobelny, C. Moskowitz, and S.H. Talisa, Electromagnetic design of high-temperature superconducting microwave filters, Int J Microwave and Millimeter-Wave CAE 5 (1995), 331-343.

16. J.W. Bandler, Q.S. Cheng, N.K. Nikolova, and M.A. Ismail, Implicit space mapping optimization exploiting preassigned parameters, IEEE Trans Microwave Theory Tech 52 (2004), 378-385.

17. S. Koziel, J.W. Bandler, A.S. Mohamed, and K. Madsen, Enhanced surrogate models for statistical design exploiting space mapping technology, 2005 IEEE MTT-S Int Microwave Symp, Long Beach, CA, 2005, 1609-1612.

\section{BIOGRAPHIES}

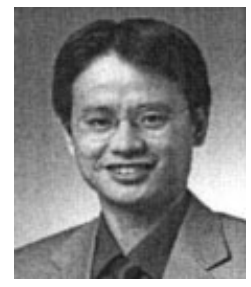

Qingsha (Shasha) Cheng was born in Chongqing, China. He received the B.Eng. degree and M.Eng. degrees in automation from Chongqing University, Chongqing, China, in 1995 and 1998, respectively. He received a Ph.D. degree at McMaster University, Hamilton, Ontario, Canada, in 2004. Currently he is a Postdoctoral Fellow in the Simulation Optimization Systems Research Laboratory, Department of Electrical and Computer Engineering, McMaster University. In September 1998, he joined the Department of Computer Science and Technology at Peking University, Beijing, China. In September 1999, he joined the Simulation Optimization Systems Research Laboratory in the Department of Electrical and Computer Engineering at McMaster University. His research interests are surrogate modeling, computer-aided design, modeling of microwave devices, software design technology and methodologies for microwave CAD.

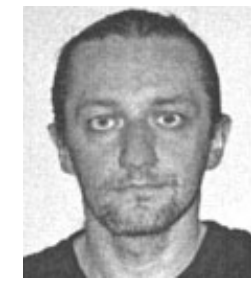

Slawomir Koziel was born in Poland in 1970. He received the M.Sc. and Ph.D. (with honors) degrees in electronic engineering from Gdansk University of Technology, Poland, in 1995 and 2000, respectively. He also received an M.Sc. degree in theoretical physics and in mathematics from the University of Gdansk, Poland, in 2000 and 2002, respectively, as well as the Ph.D. in mathematics (with honors) also from the University of Gdansk, in 2003. He is currently a Postdoctoral Fellow in the Simulation Optimization Systems Research Laboratory, Department of Electrical and Computer Engineering, McMaster University, Canada. He has published more than 80 papers. His research interests include space mapping based optimization and modeling, circuit theory, analog signal processing, active filter design, evolutionary computation, and numerical analysis. 


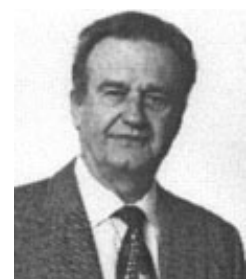

John W. Bandler was born in Jerusalem, on November 9, 1941. He studied at Imperial College of Science and Technology, London, England, from 1960 to 1966 . He received B.Sc.(Eng.), Ph.D., and D.Sc.(Eng.) degrees from the University of London, London, England, in 1963, 1967, and 1976, respectively. He joined Mullard Research Laboratories, Redhill, Surrey, England in 1966. From 1967 to 1969 he was a Postdoctorate Fellow and Sessional Lecturer at the University of Manitoba, Winnipeg, Canada. He joined McMaster University, Hamilton, Canada, in 1969. He has served as Chair of the Department of Electrical Engineering and Dean of the Faculty of Engineering. He is currently Professor Emeritus in Electrical and Computer Engineering, directing research in the Simulation Optimization Systems Research Laboratory. He was a member of the Micronet Network of Centres of Excellence. He was President of Optimization Systems Associates Inc. (OSA), which he founded in 1983, until November 20,1997, the date of acquisition of OSA by Hewlett-Packard Company (HP). OSA implemented a first-generation yield-driven microwave CAD capability for Raytheon in 1985 , followed by further innovations in linear and nonlinear microwave CAD technology for the Raytheon/Texas Instruments Joint Venture MIMIC Program. OSA introduced the CAE systems RoMPE $^{\mathrm{TM}}$ in 1988, HarPETM in 1989, OSA90 ${ }^{\mathrm{TM}}$ and OSA90/ hope $^{\mathrm{TM}}$ in 1991, Empipe ${ }^{\mathrm{TM}}$ in 1992, and Empipe3D ${ }^{\mathrm{TM}}$ and EmpipeExpress $^{\mathrm{TM}}$ in 1996. OSA created the product empath $^{\mathrm{TM}}$ in 1996 which was marketed by Sonnet Software, Inc. He is President of Bandler Corporation, which he founded in 1997. He was an Associate Editor of the IEEE Transactions on Microwave Theory and Techniques (1969-1974), and has continued serving as a member of the Editorial Board. He was Guest Editor of the Special Issue of the IEEE Transactions on Microwave Theory and Techniques on Computer-Oriented Microwave Practices (1974) and Guest CoEditor of the Special Issue of the IEEE Transactions on Microwave Theory and Techniques on Process-Oriented Microwave CAD and
Modeling (1992). He joined the Editorial Boards of the International Journal of Numerical Modelling in 1987, the International Journal of Microwave and Millimeterwave Computer-Aided Engineering in 1989, and Optimization and Engineering in 1998. He was Guest Editor, International Journal of Microwave and Millimeter-Wave Computer-Aided Engineering, Special Issue on Optimization-Oriented Microwave CAD (1997), and Guest Editor, IEEE Transactions on Microwave Theory and Techniques, Special Issue on Automated Circuit Design Using Electromagnetic Simulators (1997). He was Guest Co-Editor, Optimization and Engineering, Special Issue on Surrogate Modelling and Space Mapping for Engineering Optimization (2001). He was Guest Co-Editor, IEEE Transactions on Microwave Theory and Techniques, Special Issue on Electromagnetics-Based Optimization of Microwave Components and Circuits (2004). He has served as Chair of the MTT-1 Technical Committee on Computer-Aided Design. He has published more than 385 papers from 1965 to 2006 . He contributed to "Modern Filter Theory and Design" (Wiley-Interscience, 1973) and to "Analog Methods for Computer-Aided Analysis and Diagnosis" (Marcel Dekker, 1988). Four of his papers have been reprinted in "Computer-Aided Filter Design (IEEE Press, 1973), one in each of "Microwave Integrated Circuits" (Artech House, 1975), "Low-Noise Microwave Transistors and Amplifiers" (IEEE Press, 1981), "Microwave Integrated Circuits" (2nd ed., Artech House, 1985), "Statistical Design of Integrated Circuits" (IEEE Press, 1987), and "Analog Fault Diagnosis" (IEEE Press, 1987). He is a Fellow of the Canadian Academy of Engineering, a Fellow of the Royal Society of Canada, a Fellow of the Institute of Electrical and Electronics Engineers, a Fellow of the Institution of Electrical Engineers (Great Britain), a Fellow of the Engineering Institute of Canada, a Member of the Association of Professional Engineers of the Province of Ontario (Canada), and a Member of the MIT Electromagnetics Academy. He received the Automatic Radio Frequency Techniques Group (ARFTG) Automated Measurements Career Award in 1994 and the IEEE MTT-S Microwave Application Award in 2004. 